\title{
Event-based control of linear hyperbolic systems of conservation laws
}

\author{
Nicolás Espitia $^{\mathrm{a}, \mathrm{b}}$, Antoine Girard ${ }^{\mathrm{c}}$, Nicolas Marchand ${ }^{\mathrm{a}, \mathrm{b}}$, Christophe Prieur ${ }^{\mathrm{a}, \mathrm{b}}$ \\ ${ }^{\text {a } C N R S, ~ G I P S A-l a b, ~} 11$ rue des Mathématiques, BP 46, 38402 Grenoble, France. \\ ${ }^{\mathrm{b}}$ Université Grenoble Alpes, GIPSA-lab, BP 46, 38402 Grenoble, France. \\ ${ }^{\mathrm{c}}$ Laboratoire des signaux et systèmes (L2S), CNRS, CentraleSupélec, Université Paris-Sud, Université Paris-Saclay, 3, rue \\ Joliot-Curie, 91192 Gif-sur-Yvette, cedex, France
}

\begin{abstract}
In this article, we introduce event-based boundary controls for 1-dimensional linear hyperbolic systems of conservation laws. Inspired by event-triggered controls developed for finite-dimensional systems, an extension to the infinite dimensional case by means of Lyapunov techniques, is studied. The main contribution of the paper lies in the definition of two event-triggering conditions, by which global exponential stability and well-posedness of the system under investigation is achieved. Some numerical simulations are performed for the control of a system describing traffic flow on a roundabout.
\end{abstract}

Key words: Linear hyperbolic systems; Lyapunov techniques; Triggering conditions; Piecewise continuous functions.

\section{Introduction}

Event-based control is a computer control strategy which aims to use communications and computational resources efficiently by updating control inputs aperiodically, only when needed. Several works have been developed in this area for finite dimensional networked control systems (see for instance the seminal work $[1,36]$ or the most recent ones $[18,27]$ and the references therein). Two components are essential in the framework of eventbased control. The first one is a feedback control law which has been designed to stabilize the system. The second one is a triggering strategy which determines the time instants when the control needs to be updated. Usually, the triggering strategy guarantees that a Lyapunov function decreases strictly either by using an Input-toState Stability (ISS) property [34] or by working directly on the time derivative of the Lyapunov function [24]. Besides the interest of reducing communication and computational loads, event-based control is also known as a rigorous way to implement digitally continuous time controllers. In this work, such control strategies were de-

\footnotetext{
* This work has been partially supported by the LabEx PERSYVAL-Lab (ANR-11-LABX-0025-01)

Email addresses: nicolas.espitia-hoyos@gipsa-lab.fr (Nicolás Espitia),

antoine.girard@12s . centralesupelec.fr (Antoine

Girard), nicolas.marchand@gipsa-lab.fr (Nicolas

Marchand), christophe.prieur@gipsa-lab.fr

(Christophe Prieur).
}

veloped for a class of infinite-dimensional systems of conservation laws, provided by linear hyperbolic partial differential equations (PDEs).

Hyperbolic systems of conservation laws stand out as having important applications in the modelling and control of physical networks: hydraulic [2], road traffic [4], gas pipeline networks [16], to name a few. Stability analysis and stabilization of such systems have attracted a lot of attention in the last decade. Two ways of acting on these systems exist: boundary and in domain control. For boundary control, backstepping [19,7] and Lyapunov techniques $[6,14,29,25]$ are the most commonly used. Several applications, in which control actions are on the boundary, can be found for instance in $[3,5,11,30]$ where the exponential stability of steady-states depends on the dissipativity of the boundary conditions. This paper focuses on boundary control using Lyapunov techniques where such a dissipativity property is an important issue to be taken into account.

The design of event-based control strategies for distributed parameter systems is rarely treated in the literature. Extending existing results for ordinary differential equations (ODEs) to time-delay systems is proposed in [12]; however, this is quite far from the problem addressed in this paper. For parabolic PDEs, event-based control strategies are considered in [31] and [35]. Many difficulties that arise in the context of event-based control are due to the introduction of discontinuities when updating the control. Discontinuous output feedback con- 
trollers for infinite dimensional systems have been studied, for instance in [26], where unit feedback controller and in turn global asymptotic stabilization are considered. Although, the framework of switched hyperbolic systems $[17,28,20]$ is highly inspiring -especially the last work- for dealing with the well-posedness of the closedloop solution of such systems under event-based control strategies. The main difference of [20] with respect to the current work is that in [20], no boundary control inputs are considered but rather switching boundary conditions governed by a switching signal, given as a output feedback, that imposes the mode that the system must evolve.

The main contribution of this paper is to propose a rigorous framework for event-based control of linear hyperbolic systems of conservation laws, as well as two eventbased stabilization strategies based on the two aforementioned main triggering strategies developed for systems described by ODEs called ISS event-based stabilization and $D^{+} V$ event-based stabilization in the sequel. The notion of existence and uniqueness of the solution is treated. It is also established that the number of events in a bounded time interval is necessarily bounded avoiding the well known Zeno phenomena. To the author's knowledge, this work is the first contribution to eventbased control for hyperbolic PDE systems proposed in the literature. For PDEs, a well known approach for digital controller synthesis relies on numerical approximations by discretizing the space in order to get an ODE (see e.g. [10]) on which finite dimensional approaches can be applied. In this work, the method is completely different and adresses directly the boundary control without model reduction and the sampling in time of continuous controllers so that implementations on a digital platform may be carried out in an aperiodic fashion.

This paper is organized as follows. In Section 2, we introduce the class of linear hyperbolic system of conservation laws, and a sufficient condition for the existence of the solution is discussed. Section 3 contains the main results that are two strategies for event-based control. The existence and uniqueness of the solution as well as the stability of the closed loop systems are discussed. Section 4 provides a numerical example to illustrate the main results and to compare the two control strategies. Finally, conclusions and perspectives are given in Section 5 .

Preliminary definitions and notation. $\mathbb{R}^{+}$will denote the set of nonnegative real numbers. For any vector $v$, its $\mathrm{d}^{\text {th }}$ component will be denoted $v_{d}$. Given a matrix $A$, its transpose will be denoted $A^{T}$ and its component at row $i$ and column $j$ will be denoted by $A_{i j}$. 0 will denote the zero matrix of suitable dimension.

The usual Euclidean norm in $\mathbb{R}^{n}$ is denoted by $|\cdot|$ and the associated matrix norm is denoted $\|\cdot\|$. The set of all functions $\phi:[0,1] \rightarrow \mathbb{R}^{n}$ such that $\int_{0}^{1}|\phi(x)|^{2}<\infty$ is denoted by $L^{2}\left([0,1], \mathbb{R}^{n}\right)$ that is equipped with the norm $\|\cdot\|_{L^{2}\left([0,1], \mathbb{R}^{n}\right)}$. The restriction of a function $y: I \rightarrow J$ on an open interval $\left(x_{1}, x_{2}\right) \subset I$ is denoted by $\left.y\right|_{\left(x_{1}, x_{2}\right)}$. Given an interval $I \subseteq \mathbb{R}$ and a set $J \subseteq \mathbb{R}^{n}$ for some $n \geq 1$, a piecewise left-continuous function (resp. a piecewise right-continuous function) $y: I \rightarrow J$ is a function continuous on each closed interval subset of $I$ except maybe on a finite number of points $x_{0}<x_{1}<$ $\ldots<x_{p}$ such that for all $l \in\{0, . ., p-1\}$ there exists $y_{l}$ continuous on $\left[x_{l}, x_{l+1}\right]$ and $y_{l \mid\left(x_{l}, x_{l+1}\right)}=y_{\mid\left(x_{l}, x_{l+1}\right)}$. Moreover, at the points $x_{0}, \cdots, x_{p}$ the function is continuous from the left (resp. from the right). The set of all piecewise left-continuous functions (resp. piecewise right-continuous functions) is denoted by $\mathcal{C}_{\text {lpw }}(I, J)$ (resp. $\left.\mathcal{C}_{r p w}(I, J)\right)$. In addition, we have the following inclusions $\mathcal{C}_{\text {lpw }}\left([0,1], \mathbb{R}^{n}\right), \mathcal{C}_{\text {rpw }}\left([0,1], \mathbb{R}^{n}\right) \subset L^{2}\left([0,1], \mathbb{R}^{n}\right)$.

\section{Linear Hyperbolic Systems}

Let us consider the linear hyperbolic system of conservation laws (given in Riemann coordinates):

$$
\partial_{t} y(t, x)+\Lambda \partial_{x} y(t, x)=0 \quad x \in[0,1], t \in \mathbb{R}^{+}
$$

where $y: \mathbb{R}^{+} \times[0,1] \rightarrow \mathbb{R}^{n}, \Lambda$ is a diagonal matrix in $\mathbb{R}^{n \times n}$ such that $\Lambda=\operatorname{diag}\left(\lambda_{1}, \cdots, \lambda_{n}\right)$ with $0<\lambda_{1}<$ $\lambda_{2}<\cdots<\lambda_{n}$. We consider the following boundary condition:

$$
y(t, 0)=H y(t, 1)+B u(t), \quad t \in \mathbb{R}^{+}
$$

where $H \in \mathbb{R}^{n \times n}, B \in \mathbb{R}^{n \times m}$ and $u: \mathbb{R}^{+} \rightarrow \mathbb{R}^{m}$.

In addition to the partial differential equation (1) and the boundary condition (2), we consider the initial condition given by

$$
y(0, x)=y^{0}(x), \quad x \in[0,1]
$$

where $y^{0} \in \mathcal{C}_{\text {lpw }}\left([0,1], \mathbb{R}^{n}\right)$.

Remark 1. The results in this paper can be extended to first order linear hyperbolic systems with both negative and positive speeds $\left(\lambda_{1}<\cdots \lambda_{m}<0<\lambda_{m+1}<\cdots<\right.$ $\left.\lambda_{n}\right)$ by defining the state description $y=\left[\begin{array}{ll}y_{-} & y_{+}\end{array}\right]^{T}$, where $y_{-} \in \mathbb{R}^{m}$ and $y_{+} \in \mathbb{R}^{n-m}$, and applying the change of variable $\tilde{y}(t, x)=\left[y_{-}(t, 1-x) \quad y_{+}(t, x)\right]^{T}$.

We shall consider possibly discontinuous inputs $u \in$ $\mathcal{C}_{\text {rpw }}\left(\mathbb{R}^{+}, \mathbb{R}^{m}\right)$, therefore solutions of (1)-(3) may not be differentiable everywhere. Thus, we introduce a notion of weak solutions (generalized ones) (in Subsection 2.1) as well as a sufficient condition for the existence and uniqueness of the solution for a class of discontinuous initial conditions and feedback laws (in Subsection 2.2).

\subsection{Solution of the system}

We consider solutions of (1)-(3) in the sense of characteristics $[22]$. For each component $y_{d}$ of $(1)$, one can define the characteristic curve solution of the differential equation $\dot{x}(t)=\lambda_{d}$ which is rewritten as $x(t)=x_{0}+\lambda_{d} t$. By doing this, we obtain the following definition (see [20, Definition 4] for a more general case):

Definition 1. Let $y^{0} \in \mathcal{C}_{\text {lpw }}\left([0,1], \mathbb{R}^{n}\right)$ and $u \in$ $\mathcal{C}_{\text {rpw }}\left(\mathbb{R}^{+}, \mathbb{R}^{m}\right)$. A solution to (1)-(3) is a function $y$ : 
$\mathbb{R}^{+} \times[0,1] \rightarrow \mathbb{R}^{n}$ such that, for all $t$ in $\mathbb{R}^{+}$and $x_{0} \in$ $\left[-\lambda_{d} t, 1-\lambda_{d} t\right]$

$$
\frac{d}{d t} y_{d}\left(t, x_{0}+\lambda_{d} t\right)=0
$$

with the initial condition

$$
y_{d}(0, x)=y_{d}^{0}(x), \quad \forall x \in[0,1]
$$

and the boundary condition

$$
y_{d}(t, 0)=\sum_{j=1}^{n} H_{d j} y_{j}(t, 1)+\sum_{j=1}^{m} B_{d j} u_{j}(t), \quad \forall t \in \mathbb{R}^{+}
$$

for all $d=1, . ., n$.

Note that for classical differentiable solutions, (1)-(3) are equivalent to (4)-(6) and note that $y$ does not need to be differentiable nor continuous in general but only differentiable along the characteristics as given by (4).

In this paper, we assume that the linear hyperbolic system is only observed at the point $x=1$ at any time. Therefore we define the output function as follows:

$$
z(t)=y(t, 1)
$$

\subsection{A sufficient condition for the existence and unique- ness of the solution}

Now that solutions intended in this paper are properly defined, we will consider the following causality assumption:

Assumption 1. Let $\varphi$ be an operator from $\mathcal{C}_{\text {rpw }}\left(\mathbb{R}^{+}, \mathbb{R}^{n}\right)$ to $\mathcal{C}_{\text {rpw }}\left(\mathbb{R}^{+}, \mathbb{R}^{m}\right)$ satisfying the following causality property: for all $s$ in $\mathbb{R}^{+}$, for all $z, z^{*} \in \mathcal{C}_{\text {rpw }}\left(\mathbb{R}^{+}, \mathbb{R}^{n}\right)$

$$
\left(\forall t \in[0, s], z(t)=z^{*}(t)\right) \Longrightarrow\left(\forall t \in[0, s], u(t)=u^{*}(t)\right)
$$

where $u=\varphi(z)$ and $u^{*}=\varphi\left(z^{*}\right)$.

This assumption enables us to state the following result on existence of solutions:

Proposition 1. Let $\varphi$ satisfy Assumption 1 and $y^{0} \in$ $\mathcal{C}_{\text {lpw }}\left([0,1], \mathbb{R}^{n}\right)$. Then, there exists a unique solution to the closed-loop system (1)-(3) with controller $u=\varphi(z)$ where $z$ is defined by (7). Moreover, for all $t \in \mathbb{R}^{+}$ $y(t, \cdot) \in \mathcal{C}_{\text {lpw }}\left([0,1], \mathbb{R}^{n}\right)$ and for all $x \in[0,1] y(\cdot, x) \in$ $\mathcal{C}_{\text {rpw }}\left(\mathbb{R}^{+}, \mathbb{R}^{n}\right)$.

Proof. Let us consider $\bar{\lambda}=\max _{1 \leq i \leq n}\left\{\lambda_{i}\right\}$ and let $\delta=$ $1 / \bar{\lambda}$ be the minimum time for a characteristic, with velocity $\bar{\lambda}$, to cross the spatial domain $[0,1]$. For $p \in \mathbb{N}$, let $\Delta_{p} \subset \mathbb{R}^{+}$be defined by $\Delta_{p}=[p \delta,(p+1) \delta]$. We will proceed by induction over the interval $\Delta_{p}$ with the following induction property:

- $y$ is defined on $\Delta_{p} \times[0,1]$;

- $y(\cdot, x) \in \mathcal{C}_{r p w}\left(\Delta_{p}, \mathbb{R}^{n}\right)$;

- $y(t, \cdot) \in \mathcal{C}_{l p w}\left([0,1], \mathbb{R}^{n}\right)$.
Let us consider, for $p=0$, the interval $\Delta_{0}=[0, \delta]$. We first check that $y$ given, for all $(t, x) \in \Delta_{0} \times[0,1]$ and $d \in\{1, \ldots, n\}$, by

$$
y_{d}(t, x)= \begin{cases}y_{d}\left(t-\frac{x}{\lambda_{d}}, 0\right), & \text { if } \lambda_{d} t \geq x \\ y_{d}^{0}\left(x-\lambda_{d} t\right), & \text { if } \lambda_{d} t<x\end{cases}
$$

is the solution to system (1)-(3). Equation (8) can be written in an equivalent way; that is, for all $t$ in $\Delta_{0}, x_{0}$ in $\left[-\lambda_{d} t, 1-\lambda_{d} t\right]$, the solution at each component is given by

$$
y_{d}\left(t, x_{0}+\lambda_{d} t\right)= \begin{cases}y_{d}\left(\frac{-x_{0}}{\lambda_{d}}, 0\right), & \text { if } x_{0} \leq 0 \\ y_{d}^{0}\left(x_{0}\right), & \text { if } x_{0}>0\end{cases}
$$

Since (9) does not depend on $t$, it is clear that $\frac{d}{d t} y_{d}\left(t, x_{0}+\right.$ $\left.\lambda_{d} t\right)$ satisfies Definition 1, and hence $y$ is solution to (1)(3). Conversely, $\frac{d}{d t} y_{d}\left(t, x_{0}+\lambda_{d} t\right)=0$ implies $f\left(x_{0}\right)=$ $y_{d}\left(t, x_{0}+\lambda_{d} t\right)$ for all $t \in[0, \delta]$ and $x_{0} \in\left[-\lambda_{d} t, 1-\lambda_{d} t\right]$. In particular, by setting $t=0$, one gets

$$
f\left(x_{0}\right)=y_{d}\left(0, x_{0}\right) \text { if } x_{0}>0
$$

On the other hand, by setting $t=\frac{-x_{0}}{\lambda_{d}}$,

$$
f\left(x_{0}\right)=y_{d}\left(-\frac{x_{0}}{\lambda_{d}}, 0\right) \text { if } x_{0} \leq 0
$$

Therefore, we have obtained equation (9) and then, shown that it is the unique solution to (1)-(3) in the sense of characteristics on $\Delta_{0} \times[0,1]$. Furthermore, when $x=1, \lambda_{d} t<1$ and for all $t \in \Delta_{0}$, one gets

$$
z_{d}(t)=y_{d}(t, 1)=y_{d}^{0}\left(1-\lambda_{d} t\right)
$$

Since $z$ is well defined on $\Delta_{0}$, using the causality property, one can claim that $u$ is well defined on $\Delta_{0}$. In addition, using (10) and boundary condition (6), (8) can be rewritten as follows,

$y_{d}(t, x)= \begin{cases}\sum_{j=1}^{n} H_{d j} z_{j}\left(t-\frac{x}{\lambda_{d}}\right)+\sum_{j=1}^{m} B_{d j} u_{j}\left(t-\frac{x}{\lambda_{d}}\right), & \text { if } \lambda_{d} t \geq x \\ y_{d}^{0}\left(x-\lambda_{d} t\right), & \text { if } \lambda_{d} t<x\end{cases}$

It is worth remarking that $z_{j}\left(t-\frac{x}{\lambda_{d}}\right)=y_{j}^{0}\left(1-\lambda_{d} t+x\right)$. Then, it is proved that $y_{d}$ depends uniquely on $y_{d}^{0}$ on $\Delta_{0} \times[0,1]$.

Since $y^{0}$ belongs to $\mathcal{C}_{\text {lpw }}\left([0,1], \mathbb{R}^{n}\right), z$ belongs to $\mathcal{C}_{\text {rpw }}\left(\Delta_{0}, \mathbb{R}^{n}\right)$. Therefore, by Assumption $1, u$ belongs to $\mathcal{C}_{\text {rpw }}\left(\Delta_{0}, \mathbb{R}^{m}\right)$. It follows then, from $(11)$, that $y$ is defined on $\Delta_{0} \times[0,1]$. Moreover,

- $z_{j}\left(t-\frac{x}{\lambda_{d}}\right)$ belongs to $\mathcal{C}_{r p w}$ with respect to $t$ and belongs to $\mathcal{C}_{l p w}$ with respect to $x$ due to the opposite sign in the argument;

- $u_{j}\left(t-\frac{x}{\lambda_{d}}\right)$ belongs to $\mathcal{C}_{r p w}$ with respect to $t$ and belongs to $\mathcal{C}_{l p w}$ with respect to $x$ due to the opposite sign in the argument;

- $y_{d}^{0}\left(x-\lambda_{d} t\right)$ belongs to $\mathcal{C}_{l p w}$ with respect to $x$ and belongs to $\mathcal{C}_{r p w}$ with respect to $t$ due to the opposite sign in the argument. 
It follows from $(11)$ that $y(t, \cdot) \in \mathcal{C}_{\text {lpw }}\left([0,1], \mathbb{R}^{n}\right)$ for all $t$ in $\Delta_{0}$ and that $y(\cdot, x) \in \mathcal{C}_{r p w}\left(\Delta_{0}, \mathbb{R}^{n}\right)$ for all $x \in[0,1]$. Thus, induction property holds at $p=0$.

Now, assume that induction property holds for a given $p \in \mathbb{N}$. We are now going to prove the same property for $p+1>0$. For that purpose, let us take $y((p+1) \delta, \cdot)$ as the initial condition of the system. Applying the same arguments as above; and by means of hypothesis of induction, one gets that $z \in \mathcal{C}_{r p w}\left(\Delta_{p+1}, \mathbb{R}^{n}\right), u \in \mathcal{C}_{r p w}\left(\Delta_{p+1}, \mathbb{R}^{m}\right)$. In addition, $y$ is defined on $\Delta_{p+1} \times[0,1], y(t, \cdot)$ exists for all $t$ in $\Delta_{p+1}$ and belongs to $\mathcal{C}_{l p w}\left([0,1], \mathbb{R}^{n}\right)$ and $y(\cdot, x)$ belongs to $\mathcal{C}_{r p w}\left(\Delta_{p+1}, \mathbb{R}^{n}\right)$ for every $x \in[0,1]$.

Therefore, we have proved by induction that, for each $p \in \mathbb{N}, z \in \mathcal{C}_{r p w}\left(\Delta_{p}, \mathbb{R}^{n}\right)$ and $y(t, \cdot)$ exists for all $t$ in $\Delta_{p}$, and belongs to $\mathcal{C}_{\text {lpw }}\left([0,1], \mathbb{R}^{n}\right)$ and $y(\cdot, x)$ belongs to $\mathcal{C}_{\text {rpw }}\left(\Delta_{p}, \mathbb{R}^{n}\right)$ for every $x \in[0,1]$. Thus, there exists an unique solution to the closed-loop system (1)-(3) with $u=\varphi(z)$. Hence, this concludes the proof.

\section{Event-based Stabilization}

\subsection{Some issues related to stability}

We define the notion of stability considered in the paper and state one existing result on stability of linear hyperbolic systems of conservation laws.

Definition 2. The linear hyperbolic system (1)-(3),(7) with controller $u=\varphi(z)$ is globally exponentially stable $(G E S)$ if there exist $\nu>0$ and $C>0$ such that, for every $y^{0} \in \mathcal{C}_{\text {lpw }}\left([0,1] ; \mathbb{R}^{n}\right)$, the solution satisfies, for allt in $\mathbb{R}^{+}$,

$$
\|y(t, \cdot)\|_{\left.L^{2}([0,1]) ; \mathbb{R}^{n}\right)} \leq C e^{-\nu t}\left\|y^{0}\right\|_{L^{2}\left([0,1] ; \mathbb{R}^{n}\right)}
$$

We want to point out that a particular case studied in literature (see e.g. [8]) is when $\varphi$ is given by $u=\varphi_{0}(z)$ as $u(t)=K z(t)$. This corresponds to continuous time control for which it holds,

$$
y(t, 0)=G z(t) \quad t \in \mathbb{R}^{+}
$$

with $G=H+B K$.

The following assumption is stated in [5] as a sufficient condition, usually called dissipative boundary condition, which guarantees that the system (1)-(3) with boundary condition (13) is globally exponentially stable. In this paper, such a sufficient condition is assumed to be satisfied.

Assumption 2. The following inequality holds:

$$
\rho_{1}(G)=\operatorname{Inf}\left\{\left\|\Delta G \Delta^{-1}\right\| ; \Delta \in \mathcal{D}_{n,+}\right\}<1
$$

where $\|\cdot\|$ denotes the usual 2 -norm of matrices in $\mathbb{R}^{n \times n}$ and $\mathcal{D}_{n,+}$ denotes the set of diagonal matrices whose elements on the diagonal are strictly positive.

Recall the following result:
Proposition 2 ([9]). Under Assumption 2, there exist $\mu>0$, and a diagonal positive definite matrix $Q \in \mathbb{R}^{n \times n}$ (with $Q=\Lambda^{-1} \Delta^{2}$ ) such that the following matrix inequality holds

$$
G^{T} Q \Lambda G<e^{-2 \mu} Q \Lambda
$$

Then, the linear hyperbolic system (1)-(3),(7),(13) is GES and (12) holds for some $C>0$ and $\nu=\mu \underline{\lambda}$ where $\underline{\lambda}=\min _{1 \leq i \leq n}\left\{\lambda_{i}\right\}$.

Under the assumption of Proposition 2, inspired by $[9$, Theorem 1], let us recall that the function defined, for all $y(\cdot) \in L^{2}\left([0,1], \mathbb{R}^{n}\right)$, by

$$
V(y)=\int_{0}^{1} y(x)^{T} Q y(x) e^{-2 \mu x} d x
$$

satisfies, along the classical solutions of (1)-(3),(7) and (13), that

$$
\begin{array}{r}
\dot{V} \leq y^{T}(\cdot, 1)\left[G^{T} Q \Lambda G-e^{-2 \mu} Q \Lambda\right] y(\cdot, 1) \\
-2 \nu \int_{0}^{1} y^{T} Q y e^{-2 \mu x} d x
\end{array}
$$

thus in regard of (15), it is a Lyapunov function. The global exponential stability along $L^{2}$ solutions follows by density (see [9] for more details).

\subsection{ISS event-based stabilization}

We introduce in this section a first event-based control scheme for hyperbolic systems of conservation laws and discuss the existence of solutions and their stability under this control strategy. This approach relies on both the Input-to-State Stability property with respect to deviations to sampling and Lyapunov techniques. It is mainly inspired by [34] where the sampling error is restricted to satisfy a state-dependent inequality. It guarantees that the ISS-Lyapunov function is strictly decreasing. In this paper, we will seek for ISS property with respect to a deviation between the continuous controller and the eventbased controller, combined with a strict Lyapunov condition using (16).

Definition 3 (Definition of $\varphi_{1}$ ). Let $\varsigma_{1}, \kappa, \eta, \mu>0, K$ in $\mathbb{R}^{m \times n}, Q$ a diagonal positive matrix in $\mathbb{R}^{n \times n}$. Let us define $\varphi_{1}$ the operator which maps $z$ to $u$ as follows:

Let $z$ be in $\mathcal{C}_{\text {rpw }}\left(\mathbb{R}^{+}, \mathbb{R}^{n}\right)$ and let $W_{1}$ be given, at $t=\frac{1}{\underline{\lambda}}$, by

$$
W_{1}(\underline{\underline{1}})=\sum_{i=1}^{n} Q_{i i} \int_{0}^{1}\left(H_{i} z\left(t-\frac{x}{\lambda_{i}}\right)\right)^{2} e^{-2 \mu x} d x
$$

and, for all $t>\frac{1}{\underline{\lambda}}$, by

$$
W_{1}(t)=\sum_{i=1}^{n} Q_{i i} \int_{0}^{1}\left(H_{i} z\left(t-\frac{x}{\lambda_{i}}\right)+B_{i} u\left(t-\frac{x}{\lambda_{i}}\right)\right)^{2} e^{-2 \mu x} d x
$$


Let $\varepsilon_{1}(t)=\varsigma_{1} W_{1}\left(\frac{1}{\lambda}\right) e^{-\eta t}$ for all $t \geq \frac{1}{\lambda}$. If $W_{1}\left(\frac{1}{\lambda}\right)>0$, let the increasing sequence of time instants $\left(t_{k}^{u}\right)$ be defined iteratively by $t_{0}^{u}=0, t_{1}^{u}=\frac{1}{\underline{\lambda}}$, and for all $k \geq 1$,

$$
\begin{aligned}
t_{k+1}^{u}=\inf \left\{t \in \mathbb{R}^{+} \mid t>t_{k}^{u} \wedge\right. & \left\|B K\left(-z(t)+z\left(t_{k}^{u}\right)\right)\right\|^{2} \\
& \left.\geq \kappa W_{1}(t)+\varepsilon_{1}(t)\right\}
\end{aligned}
$$

If $W_{1}\left(\frac{1}{\lambda}\right)=0$, let the time instants be defined by $t_{0}^{u}=0$, $t_{1}^{u}=\frac{1}{\underline{\lambda}}$ and $t_{2}^{u}=\infty$.

Finally, let the control function, $z \mapsto \varphi_{1}(z)(t)=u(t)$, be defined by:

$$
\begin{array}{ll}
u(t)=0 & \forall t \in\left[t_{0}^{u}, t_{1}^{u}\right) \\
u(t)=K z\left(t_{k}^{u}\right) & \forall t \in\left[t_{k}^{u}, t_{k+1}^{u}\right), \quad k \geq 1
\end{array}
$$

Remark 2. The boundary condition (2) with controller $u=\varphi_{1}(z)$ as defined in Definition 3 can be rewritten as:

$$
y(t, 0)=G z(t)+d(t) \quad t \in \mathbb{R}^{+}
$$

where

$$
d(t)=B K\left(-z(t)+z\left(t_{k}^{u}\right)\right) \quad t \in\left[t_{k}^{u}, t_{k+1}^{u}\right)
$$

which can be seen as a deviation between the continuous controller $u=K z$ and the event-based controller of Definition 3. Hence, we follow the perturbed system approach as in [34], [21] and [18] that we will call in the sequel ISS event-based stabilization. The event triggering condition (19) ensures that, for all $t,\|d(t)\|^{2} \leq \kappa W_{1}(t)+\varepsilon_{1}(t)$.

In addition, we point out the possible case when $W_{1}\left(\frac{1}{\lambda}\right)=$ 0 , then $\varepsilon_{1}(t)=0$. From (17), it means that for all $i=$ $1, . ., n, z(s)=0$ with $s=t-\frac{x}{\lambda_{i}}$ for all $s \in\left[t-\frac{1}{\lambda_{i}}, t\right]$. In particular, for all $i=1, . ., n, z(s)=0$ for all $s \in\left[t-\frac{1}{\lambda}, t\right]$ which means that the system has already achieved the steady state in finite time. In that scenario, event-based stabilization would not be required.

The following proposition shows that $W_{1}$ given by (18) is an estimate of $V$.

Proposition 3. Let $y$ be a solution to (1)-(3). It holds that, for all $t \geq \frac{1}{\lambda}, V(y(t, \cdot))=W_{1}(t)$, where $W_{1}(t)$ is given by (18).

Proof. Along solutions $y$ to the system (1)-(3) and since $Q$ is diagonal, (16) gives:

$$
V(y(t, \cdot))=\sum_{i=1}^{n} Q_{i i} \int_{0}^{1} y_{i}^{2}(t, x) e^{-2 \mu x} d x
$$

Using the first line of (8), in particular for all $t \geq \frac{1}{\underline{\lambda}}$, the boundary condition (6) and output function (7) one has that $y_{i}(t, x)=y_{i}\left(t-\frac{x}{\lambda_{i}}, 0\right)=H_{i} z\left(t-\frac{x}{\lambda_{i}}\right)+B_{i} u\left(t-\frac{x}{\lambda_{i}}\right)$. Therefore, for all $t \geq \frac{1}{\underline{\lambda}}$,

$$
\begin{aligned}
V(y(t, \cdot)) & =\sum_{i=1}^{n} Q_{i i} \int_{0}^{1}\left[H_{i} z\left(t-\frac{x}{\lambda_{i}}\right)+B_{i} u\left(t-\frac{x}{\lambda_{i}}\right)\right]^{2} e^{-2 \mu x} d x \\
& =W_{1}(t)
\end{aligned}
$$

This concludes the proof.

Due to the previous proposition, $W_{1}$ can be seen as an estimation of the Lyapunov function $V$. It is based only on the measured output function and the input value. As a consequence, the triggering condition in (19) depends on the measured output function and the input value as well. In addition, it will be discussed in Subsection 3.2.1 that $\varepsilon_{1}$ is to guarantee the existence and uniqueness of the closed-loop system.

In the next section, we will prove that operator $\varphi_{1}$ satisfies Assumption 1.

\subsubsection{Existence and uniqueness of the closed-loop solu- tion}

The goal is to prove that $u=\varphi_{1}(z)$ belongs to $\mathcal{C}_{\text {rpw }}\left(\mathbb{R}^{+}, \mathbb{R}^{m}\right)$ provided $z$ is in $\mathcal{C}_{\text {rpw }}\left(\mathbb{R}^{+}, \mathbb{R}^{n}\right)$ and that $\varphi_{1}$ is a causal operator.

Lemma 1. The operator $\varphi_{1}$ considered in Definition 3 satisfies Assumption 1.

Proof. Let $z$ in $\mathcal{C}_{\text {rpw }}\left(\mathbb{R}^{+}, \mathbb{R}^{n}\right)$ and $u=\varphi_{1}(z)$ where $\varphi_{1}$ is the operator given in Definition 3 . Let $J$ be a closed interval subset of $\mathbb{R}^{+}$. By hypothesis, $z$ has a finite number of discontinuities on $J$. Let $t_{1}^{z}, \cdots, t_{M}^{z} \in J$ be the increasing sequence of these discontinuity time instants; and $t_{0}^{z}$ and $t_{M+1}^{z}$ are respectively the lower bound and the upper bound of the interval $J$. We want to prove that $u$ has a finite number of discontinuities on the time interval $\left[t_{i}^{z}, t_{i+1}^{z}\right]$, with $i \in\{0, \ldots, M\}$. If $W_{1}\left(\frac{1}{\lambda}\right)=0$, there is only at most one discontinuity which is $t_{1}^{u}=\frac{1}{\underline{\lambda}}$. If $W_{1}\left(\frac{1}{\underline{\lambda}}\right)>0$, let us remark that it is sufficient to show that there is a finite number of discontinuities on the open time interval $\left(t_{i}^{z}, t_{i+1}^{z}\right)$, with $i \in\{0, \ldots, M\}$.

Let $w_{1}^{i}(t)$ be the continuation of $B K z(t)$ on the interval $\left[t_{i}^{z}, t_{i+1}^{z}\right]$ with the left limit of $B K z(t)$ in $t_{i+1}^{z}$, that is

$$
\begin{aligned}
w_{1}^{i}(t) & =B K z(t), \quad \text { if } \quad t \in\left[t_{i}^{z}, t_{i+1}^{z}\right) \\
w_{1}^{i}\left(t_{i+1}^{z}\right) & =\lim _{t \rightarrow\left(t_{i+1}^{z}\right)^{-}} B K z(t)
\end{aligned}
$$

The definition of $\mathcal{C}_{r p w}\left(\mathbb{R}^{+}, \mathbb{R}^{n}\right)$ insures that the left limit of $B K z(t)$ exists and that $w_{1}^{i}(t)$ is continuous on the closed interval $\left[t_{i}^{z}, t_{i+1}^{z}\right]$. Therefore, it is uniformly continuous. It means that for all $\zeta>0$, there exists $\tau>0$ such that

$$
\forall t, t^{\prime} \in\left[t_{i}^{z}, t_{i+1}^{z}\right]:\left|t-t^{\prime}\right|<\tau \Rightarrow\left\|w_{1}^{i}(t)-w_{1}^{i}\left(t^{\prime}\right)\right\|^{2}<\zeta
$$


We denote $\tau_{i}$ the value of $\tau$ when $\zeta=\varepsilon_{1}\left(t_{i+1}^{z}\right)$. Let the sequence $\left(t_{k}^{u}\right)$ defined by Equation (19) in Definition 3. Assume first that there exists at least two consecutive discontinuity instants in $\left(t_{i}^{z}, t_{i+1}^{z}\right)$ and let $t_{k}^{u}$ be the first one of these instants. We will deal later on whether only one time instant exists within this interval.

Let us consider $\left\|-w_{1}^{i}(t)+w_{1}^{i}\left(t_{k}^{u}\right)\right\|^{2}$ for all $t \in\left[t_{i}^{z}, t_{i+1}^{z}\right]$ where $w^{i}$ is given in (23) and (24). Combined with (19) of Definition 3 and using the continuity of $W_{1}, \varepsilon_{1}$ and $w_{1}^{i}$, it holds at time $t=t_{k+1}^{u}$ :

$$
\left\|w_{1}^{i}\left(t_{k}^{u}\right)-w_{1}^{i}\left(t_{k+1}^{u}\right)\right\|^{2} \geq \kappa W_{1}\left(t_{k+1}^{u}\right)+\varepsilon_{1}\left(t_{k+1}^{u}\right)
$$

Using the non-negativity of $W_{1}$, the fact that $\varepsilon_{1}$ is a decreasing function, the uniform continuity argument and the definition of $\tau_{i}$, one gets

$$
\begin{aligned}
& \left\|w_{1}^{i}\left(t_{k}^{u}\right)-w_{1}^{i}\left(t_{k+1}^{u}\right)\right\|^{2} \geq \varepsilon_{1}\left(t_{k+1}^{u}\right) \geq \varepsilon_{1}\left(t_{i+1}^{z}\right) \\
& \Longrightarrow\left|t_{k}^{u}-t_{k+1}^{u}\right| \geq \tau_{i}
\end{aligned}
$$

Thus, $\tau_{i}$ gives a lower bound for the duration between two input updates, depending only on the interval $\left(t_{i}^{z}, t_{i+1}^{z}\right)$.

Finally, an upper bound for the maximal number of input updates on $\left(t_{i}^{z}, t_{i+1}^{z}\right)$ is given by:

$$
\bar{s}_{i}=\left\lfloor\frac{t_{i+1}^{z}-t_{i}^{z}}{\tau_{i}}\right\rfloor
$$

If there is at most one element of the sequence $\left(t_{k}^{u}\right)$ in $\left(t_{i}^{z}, t_{i+1}^{z}\right)$ then $\bar{s}_{i}$ can be chosen equal to 1 . To conclude, the number of discontinuities of $u$ on $J$ is bounded by $\bar{S}=\sum_{i=1}^{M} \bar{s}_{i}+M+2$ which is finite.

In addition, from (20) in Definition $3, u$ is piecewise constant, which yields $u \in \mathcal{C}_{r p w}\left(\mathbb{R}^{+}, \mathbb{R}^{m}\right)$.

Let us now prove that our operator $\varphi_{1}$ satisfies the causality property. Let $s \in \mathbb{R}^{+}$and $z, z^{*} \in \mathcal{C}_{r p w}\left(\mathbb{R}^{+}, \mathbb{R}^{n}\right)$ be given such that

$$
z(t)=z^{*}(t) \quad \forall t \in[0, s]
$$

Let $u=\varphi_{1}(z)$ and $u^{*}=\varphi_{1}\left(z^{*}\right)$. It will be shown that $u(t)=u^{*}(t)$ for all $t \in[0, s]$. Let us first consider $t \in[0, s)$. It follows that $u(t)=K z\left(t_{k}^{u}\right)$ where $t_{k}^{u}$ is the previous triggering time associated to $u$. It follows also from (25) that $z\left(t_{k}^{u}\right)=z^{*}\left(t_{k}^{u^{*}}\right)$, and $t_{k}^{u}=t_{k}^{u^{*}}$ where $t_{k}^{u^{*}}$ is the previous triggering time associated to $u^{*}$. Therefore $u(t)=K z^{*}\left(t_{k}^{u^{*}}\right)=u^{*}(t)$ for all $t \in[0, s)$. Let $W_{1}$ be defined by (18) and $W_{1}^{*}$ be defined similarly replacing $z$ and $u$ by $z^{*}$ and $u^{*}$ respectively. Therefore $W_{1}(t)=W_{1}^{*}(t)$ for all $t \in[0, s]$.

Let us consider what happens at $t=s$. Three cases are pointed out:

(1) Suppose that there is no triggering time at $t=s$. In this case, $u(s)=K z\left(t_{k}^{u}\right)$ where $t_{k}^{u}=t_{k}^{u^{*}}$ is the previous triggering time. Clearly, $u^{*}(s)=K z^{*}\left(t_{k}^{u^{*}}\right)=$ $K z\left(t_{k}^{u}\right)=u(s)$.
(2) Suposse that there is a triggering time for both $u$ and $u^{*}$, that is at $s=t_{k+1}^{u}=t_{k+1}^{u^{*}}$. Then, with (25)

$$
\begin{aligned}
u\left(t_{k+1}^{u}\right) & =K z\left(t_{k+1}^{u}\right)=K z(s) \\
& =K z^{*}(s)=K z^{*}\left(t_{k+1}^{u^{*}}\right)=u^{*}\left(t_{k+1}^{u^{*}}\right)
\end{aligned}
$$

(3) Suppose that there is a triggering time at time $s$. Assume without loss of generality that $s=t_{k+1}^{u}$. Consider $f(t)=\left\|B K\left(-z(t)+z\left(t_{k}^{u}\right)\right)\right\|^{2}-\kappa W_{1}(t)-\varepsilon_{1}(t)$ and $f^{*}(t)=\left\|B K\left(-z^{*}(t)+z^{*}\left(t_{k}^{u^{*}}\right)\right)\right\|^{2}-\kappa W_{1}^{*}(t)-$ $\varepsilon_{1}(t)$. Then there exists a sequence of time $\left(s_{n}\right)$, $s_{n} \geq s$ with $s_{n} \rightarrow s$ and $f\left(s_{n}\right) \geq 0$. Since $f$ is in $\mathcal{C}_{\text {rpw }}\left(\mathbb{R}^{+}, \mathbb{R}^{n}\right), f(s) \geq 0$. According to (25) and using $W_{1}(s)=W_{1}^{*}(s)$ it follows that $f(s)=f^{*}(s) \geq$ 0 . It means that a trigger happens at the same time for $u^{*}$ and then $t_{k+1}^{u^{*}}=t_{k+1}^{u}=s$. Consequently, one comes back to the previous case and hence $u(s)=u^{*}(s)$.

Since $u$ is in $\mathcal{C}_{\text {rpw }}\left(\mathbb{R}^{+}, \mathbb{R}^{m}\right)$ and the causality property is satisfied, Assumption 1 holds. It concludes the proof.

Combining the previous lemma with Proposition 1, we get

Corollary 1. For any $y^{0}$ in $\mathcal{C}_{\text {lpw }}\left([0,1], \mathbb{R}^{n}\right)$, there exists a unique solution to the closed-loop system (1)-(3),(7) and controller $u=\varphi_{1}(z)$.

\subsubsection{Stability of the closed-loop system}

Let us now state our first main result.

Theorem 1. Let $K$ be in $\mathbb{R}^{n \times n}$ such that Assumption 2 holds for $G=H+B K$. Let $\mu>0, Q$ a diagonal positive matrix in $\mathbb{R}^{n \times n}$ and $\nu=\mu \underline{\underline{\lambda}}$ be as in Proposition 2. Let $\sigma$ be in $(0,1)$ and $\alpha>0$ such that $(1+\alpha) G^{T} Q \Lambda G \leq$ $e^{-2 \mu} Q \Lambda$. Let $\rho$ be the largest eigenvalue of $\left(1+\frac{1}{\alpha}\right) Q \Lambda, \kappa=$ $\frac{2 \nu \sigma}{\rho}, \eta>2 \nu(1-\sigma)$ and $\varepsilon_{1}$ and $\varphi_{1}$ be given in Definition 3. Let $V$ be given by (16). Then the system (1)-(3),(7) with the controller $u=\varphi_{1}(z)$ has a unique solution and is globally exponentially stable. Moreover, it holds for all $t \geq \frac{1}{\underline{\lambda}}$

$$
D^{+} V(t) \leq-2 \nu(1-\sigma) V(t)+\rho \varepsilon_{1}(t)
$$

Proof. The existence and uniqueness of a solution to system (1)-(3),(7) with controller $u=\varphi_{1}(z)$ is given by Corollary 1.

We are here going to show that the system is globally exponentially stable. Assume for the time-being that $W_{1}\left(\frac{1}{\underline{\lambda}}\right)>0$.

First, we establish the following lemma which will be necessary for the proof of Theorem 1 .

Lemma 2. Let $y$ be a solution to (1)-(3) and let $V(y)$ be given by (16). Then, $t \mapsto V(y(t, \cdot))$ is continuous and 
right differentiable on $\mathbb{R}^{+}$and its right time-derivative is given by:

$$
\begin{array}{r}
D^{+} V=y^{T}(\cdot, 0) Q \Lambda y(\cdot, 0)-y^{T}(\cdot, 1) e^{-2 \mu} Q \Lambda y(\cdot, 1) \\
-2 \mu \int_{0}^{1} y^{T}\left(\Lambda e^{-2 \mu x} Q\right) y d x
\end{array}
$$

The proof of Lemma 2 is given in the Appendix.

Using the boundary condition (2) with $u=\varphi_{1}(z)$, we obtain from its equivalent form $(21)$ that $(27)$ can be rewritten as follows:

$$
\begin{aligned}
D^{+} V= & (G y(\cdot, 1)+d)^{T} Q \Lambda(G y(\cdot, 1)+d) \\
& -y^{T}(\cdot, 1) e^{-2 \mu} Q \Lambda y(\cdot, 1)-2 \mu \int_{0}^{1} y^{T}\left(\Lambda e^{-2 \mu x} Q\right) y d x
\end{aligned}
$$

Using the output function given by (7), it gives:

$$
\begin{aligned}
D^{+} V= & (G z)^{T} Q \Lambda G z+2(G z)^{T} Q \Lambda d+d^{T} Q \Lambda d \\
& -z^{T} e^{-2 \mu} Q \Lambda z-2 \mu \int_{0}^{1} y^{T}\left(\Lambda e^{-2 \mu x} Q\right) y d x
\end{aligned}
$$

By means of a decoupling procedure between $d$ and $z$ using the Young's inequality one gets that $(G z)^{T} Q \Lambda G z+$ $2(G z)^{T} Q \Lambda d+d^{T} Q \Lambda d \leq(1+\alpha)(G z)^{T} Q \Lambda G z+(1+$ $\left.\frac{1}{\alpha}\right) d^{T} Q \Lambda d$. Since $\alpha$ is such that $(1+\alpha) G^{T} Q \Lambda G \leq$ $e^{-2 \mu} Q \Lambda$, from (28) it follows:

$$
D^{+} V \leq-2 \mu \int_{0}^{1} y^{T} \Lambda Q y e^{-2 \mu x} d x+\left(1+\frac{1}{\alpha}\right) d^{T} Q \Lambda d
$$

Since $Q$ is diagonal positive definite, it holds $\Lambda Q \geq \underline{\lambda} Q$. Thus, taking $\nu=\mu \underline{\lambda}$, it yields,

$$
D^{+} V \leq-2 \nu V+\left(1+\frac{1}{\alpha}\right) d^{T} Q \Lambda d
$$

which can be rewritten as follows:

$$
\begin{aligned}
D^{+} V & \leq-2 \nu V+\rho\|d\|^{2} \\
& =-2 \nu(1-\sigma) V-2 \nu \sigma V+\rho\|d\|^{2}, \quad \sigma \in(0,1)
\end{aligned}
$$

For all $t \geq \frac{1}{\underline{\lambda}}$, Proposition 3 implies that

$$
D^{+} V \leq-2 \nu(1-\sigma) V-2 \nu \sigma W_{1}+\rho\|d\|^{2}
$$

In order to guarantee $D^{+} V$ is strictly negative, $-2 \nu \sigma W_{1}+\rho\|d\|^{2}$ must be strictly negative. Therefore, from the definition of $\varphi_{1}$, events are triggered so as to guarantee for all $t \geq \frac{1}{\lambda},\|d\|^{2} \leq \kappa W_{1}+\varepsilon_{1}$. Using $\kappa=\frac{2 \nu \sigma}{\rho}$, we obtain for all $t \geq \frac{1}{\lambda}$,

$$
D^{+} V(t) \leq-2 \nu(1-\sigma) V(t)+\rho \varepsilon_{1}(t)
$$

Which gives inequality (26) of Theorem 1 . Then, using the Comparison principle, one gets, for all $t \geq \frac{1}{\lambda}$,

$$
\begin{aligned}
V(y(t, \cdot)) \leq & e^{-2 \nu(1-\sigma)\left(t-\frac{1}{\lambda}\right)} V\left(y\left(\frac{1}{\underline{\lambda}}, \cdot\right)\right) \\
& +\rho \int_{\underline{\frac{1}{\lambda}}}^{t} e^{-2 \nu(1-\sigma)(t-s)} \varepsilon_{1}(s) d s
\end{aligned}
$$

and thus,

$$
\begin{aligned}
V(y(t, \cdot)) \leq & V\left(y\left(\frac{1}{\underline{\lambda}}, \cdot\right)\right) e^{-2 \nu(1-\sigma)\left(t-\frac{1}{\underline{\lambda}}\right)}+\frac{\rho \varsigma_{1} W_{1}\left(\frac{1}{\underline{\lambda}}\right)}{2 \nu(1-\sigma)-\eta} e^{-\eta t} \\
& -\frac{\rho \varsigma_{1} W_{1}\left(\frac{1}{\underline{\lambda}}\right)}{2 \nu(1-\sigma)-\eta} e^{-2 \nu(1-\sigma)\left(t-\frac{1}{\underline{\lambda}}\right)-\eta \frac{1}{\underline{\lambda}}}
\end{aligned}
$$

Select $\eta>2 \nu(1-\sigma)$. Thus, we get, for all $t \geq \frac{1}{\underline{\lambda}}$,

$$
\begin{aligned}
V(y(t, \cdot)) \leq & V\left(y\left(\frac{1}{\underline{\lambda}}, \cdot\right)\right) e^{-2 \nu(1-\sigma)\left(t-\frac{1}{\lambda}\right)} \\
& +\frac{\rho \varsigma_{1} V\left(y\left(\frac{1}{\lambda}, \cdot\right)\right)}{\eta-2 \nu(1-\sigma)} e^{-2 \nu(1-\sigma)\left(t-\frac{1}{\lambda}\right)-\eta \frac{1}{\lambda}}
\end{aligned}
$$

with $V\left(y\left(\frac{1}{\underline{\lambda}}, \cdot\right)\right)=W_{1}\left(\frac{1}{\underline{\lambda}}\right)$ due to Proposition 3 . The previous inequality holds even if $W_{1}\left(\frac{1}{\lambda}\right)=0$ since in this case $V\left(y\left(\frac{1}{\underline{\lambda}}, \cdot\right)\right)=0$ for all $t \geq \frac{1}{\underline{\lambda}}$.

Let us see what happens for all $t$ in $\left[0, \frac{1}{\lambda}\right)$ so as the right hand side of the inequality depends on the initial condition $y^{0}$. For that purpose, let us consider the following function:

$$
\mathcal{V}(y)=\int_{0}^{1} y(x)^{T} Q y(x) e^{2 \theta x} d x
$$

where $\theta>0$. In addition, from Definition $3, u=0$ for all $t \in\left[0, \frac{1}{\underline{\lambda}}\right)$. It implies that the boundary condition given by $(2)$ is $y(t, 0)=H y(t, 1)$. Computing the right timederivative of $(30)$, it yields

$$
\begin{gathered}
D^{+} \mathcal{V}(y(t, \cdot))=y^{T}(t, 1)\left[H^{T} Q \Lambda H-e^{2 \theta} Q \Lambda\right] y(t, 1) \\
+\int_{0}^{1} 2 \theta y^{T} Q \Lambda y e^{2 \theta} d x
\end{gathered}
$$

There exists $\theta \geq 0$ such that $H^{T} Q \Lambda H<e^{2 \theta} Q \Lambda$. In addition, since $Q$ is a diagonal positive matrix, $\Lambda Q \leq \bar{\lambda} Q$ holds. Therefore, $D^{+} \mathcal{V} \leq 2 \theta \bar{\lambda} \mathcal{V}$. Hence, the solution of the previous differential inequality thanks to the comparison principle satisfies $\mathcal{V}(y(t, \cdot)) \leq e^{2 \theta \bar{\lambda} t} \mathcal{V}\left(y^{0}\right)$. In particular, $\mathcal{V}\left(y\left(\frac{1}{\lambda}, \cdot\right)\right) \leq e^{2 \theta \frac{\bar{\lambda}}{\lambda}} \mathcal{V}\left(y^{0}\right)$. On the other hand, (16) and (30) imply

$$
\begin{aligned}
V(y) \leq \mathcal{V}(y) & =\int_{0}^{1} y(x)^{T} Q y(x) e^{2(\theta+\mu) x} e^{-2 \mu x} d x \\
& \leq e^{2(\theta+\mu)} V(y)
\end{aligned}
$$


and thus, one gets for all $t \in\left[0, \frac{1}{\underline{\lambda}}\right)$,

$$
V(y(t, \cdot)) \leq \mathcal{V}(y(t, \cdot)) \leq e^{2 \theta \bar{\lambda} t} \mathcal{V}\left(y^{0}\right) \leq e^{2 \theta \bar{\lambda} t} e^{2(\theta+\mu)} V\left(y^{0}\right)
$$

In particular, at $t=\frac{1}{\underline{\lambda}}$ we have,

$$
V\left(y\left(\frac{1}{\underline{\lambda}}, \cdot\right)\right) \leq e^{2 \theta \frac{\bar{\lambda}}{\lambda}} e^{2(\theta+\mu)} V\left(y^{0}\right)
$$

Replacing (31) in (29) we get for all $t \geq \frac{1}{\lambda}$,

$$
\begin{aligned}
V(y(t, \cdot)) \leq & e^{2 \theta \frac{\bar{\lambda}}{\underline{\lambda}}} e^{2(\theta+\mu)} e^{-2 \nu(1-\sigma)\left(t-\frac{1}{\underline{\lambda}}\right)} V\left(y^{0}\right) \\
& +\frac{\rho \varsigma_{1} e^{2 \theta \frac{\bar{\lambda}}{\lambda}} e^{2(\theta+\mu)} V\left(y^{0}\right)}{\eta-2 \nu(1-\sigma)} e^{-2 \nu(1-\sigma)\left(t-\frac{1}{\underline{\lambda}}\right)-\eta \frac{1}{\underline{\lambda}}}
\end{aligned}
$$

Reorganizing, we finally get,

$$
\begin{aligned}
V(y(t, \cdot)) \leq & e^{\left(2 \theta \frac{\bar{\lambda}}{\underline{\lambda}}+2(\theta+\mu)+2 \nu(1-\sigma) \frac{1}{\lambda}\right)} \\
& \times\left[1+\rho \frac{\varsigma_{1} e^{-\eta \frac{1}{\lambda}}}{\eta-2 \nu(1-\sigma)}\right] e^{-2 \nu(1-\sigma) t} V\left(y^{0}\right)
\end{aligned}
$$

This ends the proof of Theorem 1.

Remark 3. We emphasize again that it was crucial that $u \in \mathcal{C}_{\text {rpw }}\left(\mathbb{R}^{+}, \mathbb{R}^{m}\right)$ just to be able to apply Proposition 1 and to prove existence and uniqueness of solution.

The decreasing function $\varepsilon_{1}$ has been added within the triggering law with the aim to prove that the control value, under the triggering condition (19), has a finite number of discontinuities as it could be seen in the proof of Lemma 1.

\section{3 $D^{+} V$ event-based stabilization}

Let us consider in this section an event-triggered control similar to the one proposed in [24]. This triggering condition is not based on the existence of an Input-toState stability assumption but relies on the time derivative of a Lyapunov function. This approach can also be found in an implicit form in [18] for a linear plant, in which it is required that the solution of an event-based implementation satisfies what they call weaker inequality. The control value is then only updated when such an inequality is violated. Without entering into further details about the aforementioned approaches for finite dimensional systems, we will just point out that, in this work, the weaker inequality that causes an event when violated is of the following form:

$$
D^{+} V_{e v} \leq(1-\sigma) D^{+} V_{\text {cont }}+\varepsilon, \quad \sigma \in(0,1)
$$

where $D^{+} V_{e v}$ is the right time-derivative of the Lyapunov function candidate of the system when the control is updated only on events and $D^{+} V_{\text {cont }}$ the right timederivative of the same Lyapunov function candidate but for a control that is continuously updated and $\varepsilon$ a nonnegative decreasing function of the time. Following the same idea, we will consider the Lyapunov function candidate $V$ given by (16). Since $d$ will here again denote the deviation between the continuously updated control and the event-triggered ones (as is $(22)$ ), $D^{+} V_{e v}$ will denote the right time-derivative of $V$ when $d \neq 0$ :

$$
\begin{aligned}
D^{+} V_{e v}=z^{T} & {\left[G^{T} Q \Lambda G-e^{-2 \mu} Q \Lambda\right] z+2(G z)^{T} Q \Lambda d } \\
+ & d^{T} Q \Lambda d+\int_{0}^{1} y^{T}\left(-2 \mu \Lambda e^{-2 \mu x} Q\right) y d x
\end{aligned}
$$

whereas $D^{+} V_{\text {cont }}$ will denote the right time-derivative of $V$ when $d=0$ :

$$
\begin{aligned}
D^{+} V_{\text {cont }}= & z^{T}\left[G^{T} Q \Lambda G-e^{-2 \mu} Q \Lambda\right] z \\
& +\int_{0}^{1} y^{T}\left(-2 \mu \Lambda e^{-2 \mu x} Q\right) y d x
\end{aligned}
$$

Now that the main idea has been stated, let us formulate this in a more rigorous way with the definition of the following operator that plays the same role as Definition 3 for the ISS based approach presented in Section 3.2. Before we state the definition, let us note that, using (32) and $(33), D^{+} V_{e v} \leq(1-\sigma) D^{+} V_{\text {cont }}+\varepsilon$ is equivalent to the following, for all $t \geq 0$ and $t_{k}^{u}$ to be defined later on,

$$
\begin{array}{r}
\sigma z(t)^{T}\left[G^{T} Q \Lambda G-e^{-2 \mu} Q \Lambda\right] z(t) \\
+2(G z(t))^{T} Q \Lambda B K\left(z\left(t_{k}^{u}\right)-z(t)\right) \\
+\left(B K\left(z\left(t_{k}^{u}\right)-z(t)\right)\right)^{T} Q \Lambda B K\left(z\left(t_{k}^{u}\right)-z(t)\right) \\
\leq 2 \mu \sigma \int_{0}^{1} y^{T} Q \Lambda y e^{-2 \mu x} d x+\varepsilon(t)
\end{array}
$$

Definition 4 (Definition of $\left.\varphi_{2}\right)$. Let $\varsigma_{2}, \eta>0, \sigma \in(0,1)$, $K$ in $\mathbb{R}^{m \times n}, G=H+B K$ in $\mathbb{R}^{n \times n}, D$ a diagonal positive definite matrix in $\mathbb{R}^{n \times n}, \mu>0$ such that $G^{T} D G-e^{-2 \mu} D$ is a negative symmetric matrix in $\mathbb{R}^{n \times n}, V$ given by (16) and $\varepsilon_{2}(t)=\varsigma_{2} V\left(\frac{1}{\lambda}\right) e^{-\eta t}$ for all $t \geq \frac{1}{\lambda}$. Let us define $\varphi_{2}$ the operator which maps $z$ to $u$ as follows:

Let $z$ in $\mathcal{C}_{\text {rpw }}\left(\mathbb{R}^{+}, \mathbb{R}^{n}\right)$ and the time function $W_{2}$ be defined similarly to (18), for all $t \geq \frac{1}{\underline{\lambda}}$ by:

$$
W_{2}(t)=\sum_{i=1}^{n} D_{i i} \int_{0}^{1}\left(H_{i} z\left(t-\frac{x}{\lambda_{i}}\right)+B_{i} u\left(t-\frac{x}{\lambda_{i}}\right)\right)^{2} e^{-2 \mu x} d x
$$

If $V\left(\frac{1}{\lambda}\right)>0$, let the increasing sequence of time instants $\left(t_{k}^{u}\right)$ be defined iteratively by $t_{0}^{u}=0, t_{1}^{u}=\frac{1}{\underline{\lambda}}$, and for all $k \geq 1$,

$$
\begin{array}{r}
t_{k+1}^{u}=\inf \left\{t \in \mathbb{R}^{+} \mid t>t_{k}^{u} \wedge \sigma z^{T}(t)\left[G^{T} D G-e^{-2 \mu} D\right] z(t)\right. \\
+2(G z(t))^{T} D B K\left(z\left(t_{k}^{u}\right)-z(t)\right) \\
+\left(B K\left(z\left(t_{k}^{u}\right)-z(t)\right)\right)^{T} D B K\left(z\left(t_{k}^{u}\right)-z(t)\right) \\
\left.\geq 2 \mu \sigma W_{2}(t)+\varepsilon_{2}(t)\right\}
\end{array}
$$

If $V\left(\frac{1}{\lambda}\right)=0$, let the time instants be defined by $t_{0}^{u}=0$, $t_{1}^{u}=\frac{\overline{1}}{\underline{\lambda}}$ and $t_{2}^{u}=\infty$. 
Finally, let the control function, $z \mapsto \varphi_{2}(z)(t)=u(t)$, be defined by:

$$
\begin{array}{ll}
u(t)=0 & \forall t \in\left[t_{0}^{u}, t_{1}^{u}\right) \\
u(t)=K z\left(t_{k}^{u}\right) & \forall t \in\left[t_{k}^{u}, t_{k+1}^{u}\right), \quad k \geq 1
\end{array}
$$

Remark 4. Following the same arguments as in the second part of Remark 2, whenever $V\left(\frac{1}{\lambda}\right)=0$, event-based stabilization would not be required.

o

The purpose of next two subsections is to prove that system (1)-(3),(7), with the same boundary condition (21) as before and under the event-triggered control of Definition 4 , has a unique solution for any given initial conditions and is globally exponentially stable in the sense of Definition 2.

\subsubsection{Existence and uniqueness of the closed-loop solu- tion}

As in Section 3.2.1, the goal here is to prove that $u=\varphi_{2}(z)$ belongs to $\mathcal{C}_{r p w}\left(\mathbb{R}^{+}, \mathbb{R}^{m}\right)$ whenever $z$ is in $\mathcal{C}_{r p w}\left(\mathbb{R}^{+}, \mathbb{R}^{n}\right)$ and that $\varphi_{2}$ is a causal operator.

Lemma 3. The operator $\varphi_{2}$ defined in Definition 4 satisfies Assumption 1.

Proof. The proof follows essentially the same steps as proof of Lemma 1. Let $z$ in $\mathcal{C}_{r p w}\left(\mathbb{R}^{+}, \mathbb{R}^{n}\right)$ and $u=\varphi_{2}(z)$ where $\varphi_{2}$ is the operator given in Definition 4. Let $J$ be a closed interval subset of $\mathbb{R}^{+}$. By assumption, $z$ has a finite number of discontinuities on $J$. As previously, $t_{1}^{z}, \cdots, t_{M}^{z} \in J$ will be the increasing sequence of these discontinuity time instants to which we add the the extremities $t_{0}^{z}$ and $t_{M+1}^{z}$ of the interval $J$. As in the proof of Lemma 1, our aim is to prove that $u$ has a finite number of discontinuities on the time interval $\left[t_{i}^{z}, t_{i+1}^{z}\right]$, with $i \in\{0, \ldots, M\}$ and therefore, it is sufficient to show that there is a finite number of discontinuities on the open time interval $\left(t_{i}^{z}, t_{i+1}^{z}\right)$, with $i \in\{0, \ldots, M\}$. If $V\left(\frac{1}{\underline{\lambda}}\right)=0$, there is only at most one discontinuity which is $t_{1}^{u}=\frac{1}{\underline{\lambda}}$.

If $V\left(\frac{1}{\lambda}\right)>0$, let $\gamma$ be a strictly positive real number such that the following inequality matrix holds:

$$
G^{T} D G<\frac{\sigma}{\gamma+\sigma} e^{-2 \mu} D
$$

Note that taking $\gamma$ sufficiently small, this inequality can always be satisfied. Let $w_{2} \in \mathcal{C}_{r p w}\left(\mathbb{R}^{+}, \mathbb{R}^{n}\right)$ be defined by:

$$
w_{2}(t):=\sqrt{D} B K z(t)
$$

where $D$ is as in Definition 4 (since $D$ is a diagonal positive definite matrix, $D$ has an unique positive-definite diagonal square root matrix, denoted $\sqrt{D}$, whose diagonal elements are equal to square roots of the diagonal elements of $D)$. Let $w_{2}^{i}(t)$ be the continuation of $w_{2}(t)$ given by (37), on the interval $\left[t_{i}^{z}, t_{i+1}^{z}\right]$ with the left limit of $w_{2}(t)$ in $t_{i+1}^{z}$, that is:

$$
\begin{aligned}
w_{2}^{i}(t) & =w_{2}(t), \quad \text { if } \quad t \in\left[t_{i}^{z}, t_{i+1}^{z}\right) \\
w_{2}^{i}\left(t_{i+1}^{z}\right) & =\lim _{t \rightarrow\left(t_{i+1}^{z}\right)^{-}} w_{2}(t)
\end{aligned}
$$

The definition of $\mathcal{C}_{r p w}\left(\mathbb{R}^{+}, \mathbb{R}^{n}\right)$ insures that the left limit of $w_{2}(t)$ exists and that $w_{2}^{i}(t)$ is continuous on the closed interval $\left[t_{i}^{z}, t_{i+1}^{z}\right]$. Therefore, it is uniformly continuous. It means that for all $\zeta>0$, there exists $\tau>0$ such that

$$
\forall t, t^{\prime} \in\left[t_{i}^{z}, t_{i+1}^{z}\right]:\left|t-t^{\prime}\right|<\tau \Rightarrow\left\|w_{2}^{i}(t)-w_{2}^{i}\left(t^{\prime}\right)\right\|^{2}<\zeta
$$

As in the proof of Lemma 1, we denote $\tau_{i}$ the value of $\tau$ when $\zeta=\frac{\gamma}{\gamma+1} \varepsilon_{2}\left(t_{i+1}^{z}\right)$. Here again, we assume first that there are at least two consecutive discontinuity instants in $\left(t_{i}^{z}, t_{i+1}^{z}\right)$ and let $t_{k}^{u}$ be the first one.

Let us consider the triggering condition (34) in Definition 4 and using the continuity of $W_{2}, \varepsilon_{2}$ and $w_{2}^{i}$, it holds at time $t=t_{k+1}^{u}$ :

$$
\begin{array}{r}
\sigma z^{T}\left(t_{k+1}^{u}\right)\left[G^{T} D G-e^{-2 \mu} D\right] z\left(t_{k+1}^{u}\right) \\
+2\left(G z\left(t_{k+1}^{u}\right)\right)^{T} D B K\left(z\left(t_{k}^{u}\right)-z\left(t_{k+1}^{u}\right)\right) \\
+\left(B K\left(z\left(t_{k}^{u}\right)-z\left(t_{k+1}^{u}\right)\right)\right)^{T} D B K\left(z\left(t_{k}^{u}\right)-z\left(t_{k+1}^{u}\right)\right) \\
\geq 2 \mu \sigma W_{2}\left(t_{k+1}^{u}\right)+\varepsilon_{2}\left(t_{k+1}^{u}\right)
\end{array}
$$

Using the Young's inequality, one has for $\gamma$ as in (36):

$$
\begin{array}{r}
z^{T}\left(t_{k+1}^{u}\right)\left[(\gamma+\sigma) G^{T} D G-\sigma e^{-2 \mu} D\right] z\left(t_{k+1}^{u}\right) \\
+\left(1+\frac{1}{\gamma}\right)\left(B K\left(z\left(t_{k}^{u}\right)-z\left(t_{k+1}^{u}\right)\right)\right)^{T} D B K\left(z\left(t_{k}^{u}\right)-z\left(t_{k+1}^{u}\right)\right) \\
\geq 2 \mu \sigma W_{2}\left(t_{k+1}^{u}\right)+\varepsilon_{2}\left(t_{k+1}^{u}\right)
\end{array}
$$

Knowing that $\left(B K\left(z\left(t_{k}^{u}\right)-z\left(t_{k+1}^{u}\right)\right)\right)^{T} D B K\left(z\left(t_{k}^{u}\right)-\right.$ $\left.z\left(t_{k+1}^{u}\right)\right)=\left\|\sqrt{D} B K\left(z\left(t_{k}^{u}\right)-z\left(t_{k+1}^{u}\right)\right)\right\|^{2}$ and according to (38) and (39), one gets

$$
\begin{aligned}
& \left\|w_{2}^{i}\left(t_{k}^{u}\right)-w_{2}^{i}\left(t_{k+1}^{u}\right)\right\|^{2} \geq \\
& \frac{\gamma}{1+\gamma}\left(-z^{T}\left(t_{k+1}^{u}\right)\left[(\gamma+\sigma) G^{T} D G-\sigma e^{-2 \mu} D\right] z\left(t_{k+1}^{u}\right)\right. \\
& \left.\quad+2 \mu \sigma W_{2}\left(t_{k+1}^{u}\right)+\varepsilon_{2}\left(t_{k+1}^{u}\right)\right)
\end{aligned}
$$

Moreover, using the non-negativity of $W_{2}$ and $-z^{T}\left(t_{k+1}^{u}\right)\left[(\gamma+\sigma) G^{T} D G-\sigma e^{-2 \mu} D\right] z\left(t_{k+1}^{u}\right)$, the fact that $\varepsilon_{2}$ is a decreasing function, the uniform continuity argument and the definition of $\tau_{i}$, one gets

$$
\begin{aligned}
& \left\|w_{2}^{i}\left(t_{k+1}^{u}\right)-w_{2}^{i}\left(t_{k}^{u}\right)\right\|^{2} \geq \frac{\gamma}{1+\gamma} \varepsilon_{2}\left(t_{k+1}^{u}\right) \geq \frac{\gamma}{1+\gamma} \varepsilon_{2}\left(t_{i+1}^{z}\right) \\
& \quad \Longrightarrow\left|t_{k}^{u}-t_{k+1}^{u}\right| \geq \tau_{i}
\end{aligned}
$$

Thus, $\tau_{i}$ gives a lower bound for the duration between two input updates, depending only on the interval $\left(t_{i}^{z}, t_{i+1}^{z}\right)$. The remaining part of the proof that $u$ is in $\mathcal{C}_{r p w}\left(\mathbb{R}^{+}, \mathbb{R}^{m}\right)$ follows the lines of Lemma 1 .

For proving the causality property of the operator $\varphi_{2}$, it is sufficient to follow the same steps as in 
proof of Lemma 1 but considering, at the final step of the proof, $f(t)=\sigma z^{T}(t)\left[G^{T} D G-e^{-2 \mu} D\right] z(t)+$ $2(G z(t))^{T} D B K\left(z\left(t_{k}^{u}\right)-z(t)\right)+\left(B K\left(z\left(t_{k}^{u}\right)-\right.\right.$ $z(t)))^{T} D B K\left(z\left(t_{k}^{u}\right)-z(t)\right)-2 \mu \sigma W_{2}(t)-\varepsilon_{2}(t)$ and $f^{*}(t)=\sigma\left(z^{*}\right)^{T}(t)\left[G^{T} D G-e^{-2 \mu} D\right] z^{*}(t)+$ $2\left(G z^{*}(t)\right)^{T} D B K\left(z^{*}\left(t_{k}^{u^{*}}\right)-z^{*}(t)\right)+\left(B K\left(z^{*}\left(t_{k}^{u^{*}}\right)-\right.\right.$ $\left.\left.z^{*}(t)\right)\right)^{T} D B K\left(z^{*}\left(t_{k}^{u^{*}}\right)-z^{*}(t)\right)-2 \mu \sigma W_{2}^{*}(t)-\varepsilon_{2}(t)$.

Since $u$ is in $\mathcal{C}_{r p w}\left(\mathbb{R}^{+}, \mathbb{R}^{m}\right)$ and the causality property is satisfied, Assumption 1 holds. It concludes the proof.

Combining the previous lemma with Proposition 1, we get

Corollary 2. For any $y^{0}$ in $\mathcal{C}_{\text {lpw }}\left([0,1], \mathbb{R}^{n}\right)$, there exists a unique solution to the closed-loop system (1)-(3),(7) and controller $u=\varphi_{2}(z)$.

\subsubsection{Stability of the closed-loop system}

Let us now state our second main result.

Theorem 2. Let $K$ be in $\mathbb{R}^{n \times n}$ such that Assumption 2 holds for $G=H+B K$. Let $\mu>0, Q$ a diagonal positive matrix in $\mathbb{R}^{n \times n}$ and $\nu=\mu \underline{\lambda}$ be as in Proposition 2. Let $\sigma$ be in $(0,1), \eta>0$ and $\varepsilon_{2}$ and $\varphi_{2}$ be given in Definition 4. Let $V$ be given by (16) and d given by (22). Then the system (1)-(3),(7) with the controller $u=\varphi_{2}(z)$ has a unique solution and is globally exponentially stable. Moreover, it holds for all $t \geq \frac{1}{\lambda}$,

$$
D^{+} V(t) \leq-2 \nu(1-\sigma) V(t)+\varepsilon_{2}(t)
$$

Proof. The existence and uniqueness of a solution to system (1)-(3),(7), with $u=\varphi_{2}(z)$ is given by Corollary 2 .

We are now going to show that the system is globally exponentially stable. Assume that $V\left(\frac{1}{\lambda}\right)>0$ (the other case is studied as in Theorem 1).

From the definition of $\varphi_{2}$, events are triggered so as to guarantee for all $t \geq \frac{1}{\lambda}$, $\sigma z^{T}(t)\left[G^{T} Q \Lambda G-e^{-2 \mu} Q \Lambda\right] z(t)+2(G z(t))^{T} Q \Lambda d(t) \stackrel{+}{+}$ $d^{T}(t) Q \Lambda d(t) \leq 2 \mu W_{2}(t)+\varepsilon_{2}(t)$ and therefore $D^{+} V_{e v}(t) \leq(1-\sigma) D^{+} V_{\text {cont }}(t)+\varepsilon(t)$ by taking into account that $D=Q \Lambda$ and $\varepsilon=\varepsilon_{2}$. By construction, it follows that, for all $t \geq \frac{1}{\underline{\lambda}}$,

$$
D^{+} V(t)=D^{+} V_{e v}(t) \leq(1-\sigma) D^{+} V_{\text {cont }}(t)+\varepsilon_{2}(t)
$$

Using $\Lambda Q \geq \underline{\lambda} Q$ and Proposition 2, it is clear that $D^{+} V_{\text {cont }} \leq-2 \overline{\nu V}$.

Therefore, in (41), for all $t \geq \frac{1}{\underline{\lambda}}$,

$$
D^{+} V(t) \leq-2 \nu(1-\sigma) V(t)+\varepsilon_{2}(t)
$$

Now, proceeding exactly as in the proof of Theorem 1 when using the Comparision principle and analyzing what happens for all $t \in\left[0, \frac{1}{\lambda}\right)$, the final result which proves the global exponential stability, is the following for all $t \geq \frac{1}{\lambda}$,

$$
\begin{aligned}
V(y(t, \cdot)) \leq & e^{\left(2 \theta \frac{\bar{\lambda}}{\underline{\lambda}}+2(\theta+\mu)+2 \nu(1-\sigma) \frac{1}{\lambda}\right)} \\
& \times\left[1+\frac{\varsigma_{2} e^{-\eta} \frac{1}{\underline{\lambda}}}{\eta-2 \nu(1-\sigma)}\right] e^{-2 \nu(1-\sigma) t} V\left(y^{0}\right)
\end{aligned}
$$

This ends the proof of Theorem 2 .

\subsection{Comparison between ISS event-based stabilization} and $D^{+} V$ event-based stabilization

The following proposition links the two event-based control approaches that we have proposed in this article.

Proposition 4. Let $\sigma$ be in $(0,1), \alpha, \rho, \nu, \kappa$ and $Q$ be as in Theorem 1. Let $V$ be given by $(16), D^{+} V_{\text {ev }}$ and $D^{+} V_{\text {cont }}$ be given by (32) and (33) respectively, $d$ given by (22), and $W_{1}$ as in Definition 3. The following implication holds, for all $t \geq \frac{1}{\underline{\lambda}}$,

$$
D^{+} V_{e v} \geq(1-\sigma) D^{+} V_{\text {cont }}+\varepsilon_{2} \Rightarrow\|d\|^{2} \geq \frac{2 \nu \sigma}{\rho} W_{1}+\frac{\varepsilon_{2}}{\rho}
$$

Therefore for all solutions to (1)-(3) and (7) in closedloop, respectively with controllers $u=\varphi_{1}(z)$ and $u=$ $\varphi_{2}(z)$, by selecting $\varsigma_{1}=\frac{\varsigma_{2}}{\rho}$ and having the same initial condition, the first triggering time occurs with $\varphi_{1}$ and not with $\varphi_{2}$.

Proof. It holds that $D^{+} V_{e v} \geq(1-\sigma) D^{+} V_{\text {cont }}+\varepsilon_{2}$ is equivalent to:

$$
\begin{array}{r}
\sigma z^{T}\left[G^{T} Q \Lambda G-e^{-2 \mu} Q \Lambda\right] z+2(G z)^{T} Q \Lambda d+d^{T} Q \Lambda d \\
\geq-\sigma \int_{0}^{1} y^{T}\left(-2 \mu \Lambda e^{-2 \mu x} Q\right) y d x+\varepsilon_{2}
\end{array}
$$

Using $\Lambda Q \geq \underline{\lambda} Q$ and the Young's inequality we obtain that $D^{+} V_{e v} \geq(1-\sigma) D^{+} V_{\text {cont }}+\varepsilon_{2}$ implies,

$$
\begin{aligned}
& z^{T}\left[(\sigma+\alpha) G^{T} Q \Lambda G-\sigma e^{-2 \mu} Q \Lambda\right] z+\left(1+\frac{1}{\alpha}\right) d^{T} Q \Lambda d \\
\geq & \sigma z^{T}\left[G^{T} Q \Lambda G-e^{-2 \mu} Q \Lambda\right] z+2(G z)^{T} Q \Lambda d+d^{T} Q \Lambda d \\
\geq & 2 \nu \sigma V+\varepsilon_{2}
\end{aligned}
$$

for any $\alpha>0$ and $\nu=\underline{\lambda} \mu$. Due to Assumption 14 and Proposition 2, there exists $\alpha>0$ such that $(\sigma+\alpha) G^{T} Q \Lambda G-\sigma e^{-2 \mu} Q \Lambda<0$, hence,

$D^{+} V_{e v} \geq(1-\sigma) D^{+} V_{\text {cont }}+\varepsilon_{2} \Rightarrow\left(1+\frac{1}{\alpha}\right) d^{T} Q \Lambda d \geq 2 \nu \sigma V+\varepsilon_{2}$

Taking $\rho$ as the largest eigenvalue of matrix $\left(1+\frac{1}{\alpha}\right) Q \Lambda$ one gets,

$$
D^{+} V_{e v} \geq(1-\sigma) D^{+} V_{\text {cont }}+\varepsilon_{2} \Rightarrow \rho\|d\|^{2} \geq 2 \nu \sigma V+\varepsilon_{2}
$$

Finally, using Proposition 3 , one gets, for all $t \geq \frac{1}{\underline{\lambda}}$,

$$
\|d\|^{2} \geq \kappa W_{1}+\varepsilon_{1}
$$


and thus inequality (42) holds, with $\varepsilon_{1}=\frac{\varepsilon_{2}}{\rho}$ and $\kappa=$ $\frac{2 \nu \sigma}{\rho}$.

Remark 5. One consequence of Proposition 4 is that controller $u=\varphi_{1}(z)$ generates the first triggering time before the controller $u=\varphi_{2}(z)$. Of course this does not imply that we have less triggering times with $\varphi_{2}$ than $\varphi_{1}$. However, this property and in turn, larger inter-execution times in average under $\varphi_{2}$ times, are observed on numerical simulations as we will see in the next section.

\section{Numerical simulations}

Numerical simulations were done by discretizing an example of linear hyperbolic system. For that purpose we have used a two-step variant of the Lax-Friedrichs numerical method presented in [33] and the solver on Matlab in [32]. We selected the parameters of the numerical scheme so that the Courant-Friedrich-Levy condition for the numerical stability holds. In addition, the sufficient stability condition is achieved using classical numerical tools for semi-definite programming (see e.g. Yalmip toolbox [23] with SeDuMi solver).

As a matter of example, we consider a system of $2 \times 2$ hyperbolic conservation laws describing the vehicle traffic flow on a roundabout made up of only two inputs/exits. Inspired by [3], where an example of ramp-meetering control in road traffic networks is treated, we consider the structure of Figure 1 for a network in compartmental system representation describing flows on conservative networks. Each compartment or node represents a segment of the roundabout. Based on LWR model (see e.g. [4]), let us consider the special case where the dynamic of the system is written as a set of kinematic wave equations,

$$
\partial_{t} q+C(q) \partial_{x} q=0
$$

where $q=\left[\begin{array}{ll}q_{1} & q_{2}\end{array}\right]^{T}$ is the flux. $C(q)=$ $\operatorname{diag}\left(c_{1}\left(q_{1}\right) \quad c_{2}\left(q_{2}\right)\right)$ is the matrix of characteristic velocities. The boundary conditions are

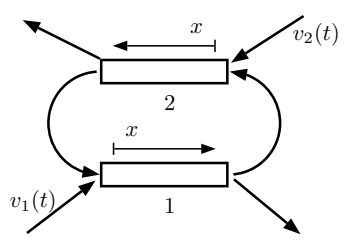

Figure 1. Network of 2 conservation laws for a roundabout.

$$
\begin{aligned}
& q_{1}(t, 0)=\gamma q_{2}(t, 1)+v_{1}(t) \\
& q_{2}(t, 0)=\beta q_{1}(t, 1)+v_{2}(t)
\end{aligned}
$$

where $\gamma$ and $\beta$ are traffic splitting factors at the two exits of the roundabout and $v_{1}(t)$ and $v_{2}(t)$ are the influxes injected from outside into compartments 1 and 2 respectively. They can be viewed as control inputs (e.g. when modulating with traffic lights). With these control actions, one intends to prevent the appearance of traffic jams on the roundabout or to achieve a desired steady-state without congestion. As in [3], we assume free-flow conditions. In that case, characteristic velocities are all positives. In addition, a steady-state for the system $\left\{q^{*}, v^{*}\right\}$ is space invariant and satisfies (44), that is $q_{1}^{*}=\gamma q_{2}^{*}+v_{1}^{*}$ and $q_{2}^{*}=\beta q_{1}^{*}+v_{2}^{*}$. We select the steadystate $\left\{q^{*}, v^{*}\right\}$ as a free-flow steady-state. We want to stabilize it under linear output feedback control of the form

$$
\begin{aligned}
& v_{1}(t)=v_{1}^{*}+k_{1}\left(q_{2}(t, 1)-q_{2}^{*}\right) \\
& v_{2}(t)=v_{2}^{*}+k_{2}\left(q_{1}(t, 1)-q_{1}^{*}\right)
\end{aligned}
$$

where $k_{1}, k_{2}$ are tuning control parameters. Defining the deviations $y=q-q^{*}$ and $u=v-v^{*}$, the linearization of system (43) at the equilibrium is given by

$$
\partial_{t} y+C\left(q^{*}\right) \partial_{x} y=0
$$

with $y=\left[\begin{array}{ll}y_{1} & y_{2}\end{array}\right]^{T}, C\left(q^{*}\right)=\operatorname{diag}\left(c_{1}\left(q_{1}^{*}\right) \quad c_{2}\left(q_{2}^{*}\right)\right)$ and the boundary condition given by

$$
y(t, 0)=H y(t, 1)+B u(t)
$$

where $H=\left(\begin{array}{ll}0 & \gamma \\ \beta & 0\end{array}\right), B=I_{2}$ and $u(t)=K y(t, 1)$ with $K=$ $\left(\begin{array}{cc}0 & k_{1} \\ k_{2} & 0\end{array}\right)$. We then perform simulations setting $c_{1}\left(q_{1}^{*}\right)=1$, $c_{2}\left(q_{2}^{*}\right)=\sqrt{2}, \gamma=0.7, \beta=0.9$. The initial condition is $y(0, x)=\left[\begin{array}{ll}4 x(x-1) & \sin (8 \pi x)\end{array}\right]^{T}$ for all $x \in[0,1]$.

It is worth remarking that due to the nature of this problem, the system in open-loop converges to the equilibrium, i.e. $\rho_{1}(H)<1$ holds, thus the system (45) in open-loop is GES. Therefore, the design of the control $u(t)$ would be rather devoted to improve the performance of the network (e.g. to accelerate the time convergence to the steady-state, by decreasing the $\rho_{1}$-norm of the boundary condition).

\subsection{Continuous stabilization: controller $u=\varphi_{0}(z)$}

The boundary condition is $y(t, 0)=H y(t, 1)+B u(t)$ where $u(t)=\varphi_{0}(z)(t)=K z(t)$ is the continuous controller acting from $t \geq \frac{1}{\underline{\lambda}}=1$. The initial condition was chosen such that the zero-order compatibility condition is satisfied i.e. $y(0,0)=(H+B K) y(0,1)$. Condition (14) holds since $\left\|\Delta_{H} H \Delta_{H}^{-1}\right\|=0.8079<1$ with $\Delta_{H}=\left(\begin{array}{cc}1.2729 & 0 \\ 0 & 0 \\ 1.1426\end{array}\right)$ and thus $\rho_{1}(H)<1$. Then, $K$ has been designed such that $\rho_{1}(G)<\rho_{1}(H)<1$ with $G=H+B K$. To be more specific, with $K=\left(\begin{array}{cc}0 & 0.3 \\ -0.9 & 0\end{array}\right)$ and $\Delta_{G}=\left(\begin{array}{cc}0.9134 & 0 \\ 0 & 1.2580\end{array}\right),\left\|\Delta_{G} G \Delta_{G}^{-1}\right\|=0.7262<1$. It implies that the closed-loop system is GES and the $\rho_{1}$-norm of the boundary condition is smaller than the open-loop case. Besides this, condition (15) in Proposition 2 was also checked getting as a result the existence of scalars $\mu=0.1, \nu=0.1$ and one symmetric matrix $Q=\left(\begin{array}{cc}0.8346 & 0 \\ 0 & 1.1191\end{array}\right)$.

\subsection{ISS event-based stabilization: controller $u=\varphi_{1}(z)$}

The boundary condition is now $y(t, 0)=H y(t, 1)+B u(t)$ where $u(t)=\varphi_{1}(z)(t)$. The parameters for the triggering 
algorithm were chosen to be $\alpha=0.5, \sigma=0.9$. Therefore, $\rho=4.7481, \kappa=0.0379$ and $\left[(1+\alpha) G^{T} Q \Lambda G-e^{-2 \mu} Q \Lambda\right]=$ $\left(\begin{array}{cc}-0.6833 & 0 \\ 0 & -0.0439\end{array}\right)$ is a symmetric negative definite matrix. Hence, Theorem 1 holds. The function $\varepsilon_{1}$ used in the triggering condition (19) is chosen to be

$$
\varepsilon_{1}(t)=\varsigma_{1} V(1) e^{-\eta t}, \quad t \in \mathbb{R}^{+}
$$

with $\eta=0.4, V(1)=0.6390$, and $\varsigma_{1}$ is such that $\varsigma_{1} V(1)=5 \times 10^{-3}$. Figure 2 shows the time evolution of

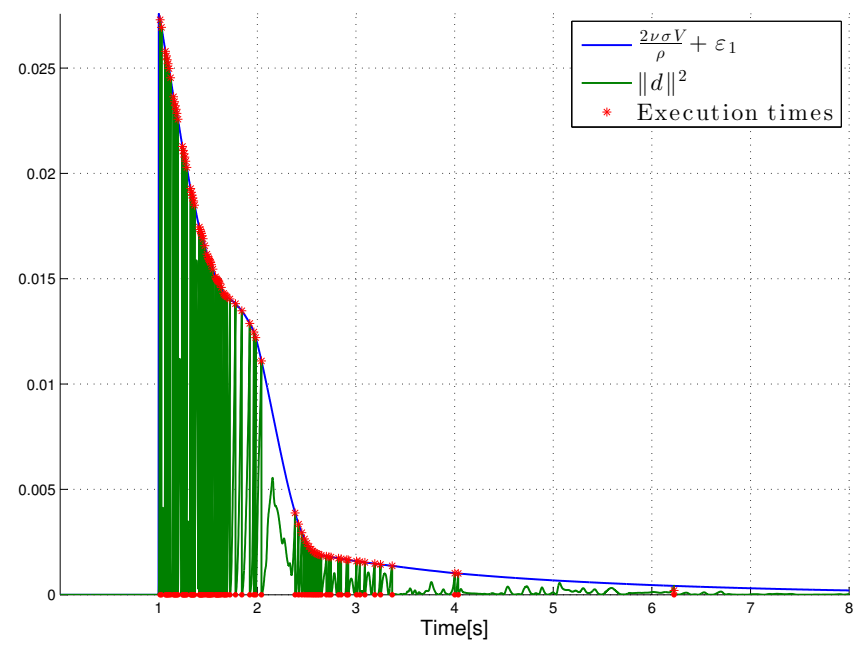

Figure 2. Trajectories involved in triggering condition (19) for controller $u=\varphi_{1}(z)$.

the functions appearing in the triggering condition (19). Once the trajectory $\|d\|^{2}$ reaches the trajectory $\kappa V+\varepsilon_{1}$, an event is generated, the control value is updated and $d$ is reset to zero. The number of events under this eventbased approach was 89 , counting them from $t \geq \frac{1}{\underline{\lambda}}=1$. It is considerable less with respect to continuous stabilization, since the number of discretization points in time is $N T=8000$ with a discretization step $\Delta t=1 \times 10^{-3}$.

\section{3 $D^{+} V$ event-based stabilization: controller $u=$ $\varphi_{2}(z)$}

The boundary condition is now $y(t, 0)=H y(t, 1)+B u(t)$ where $u(t)=\varphi_{2}(z)(t)$. The only parameter to be chosen here is $\sigma$ and it is as before, $\sigma=0.9$. The function $\varepsilon_{2}$ used in the triggering condition (34) is $\varepsilon_{2}=\rho \varepsilon_{1}$ with $\rho=4.7481$ and $\varepsilon_{1}$ given by (47). The number of events under this event-based approach was 30 , counting them from $t \geq 1$.

A comparison of the functions $V$ when stabilizing with $\varphi_{0}, \varphi_{1}$ and $\varphi_{2}$ is done as shown in Figure 3. It can be noticed that under the two event-based stabilization approaches, global asymptotic stability is achieved with different observed rates despite similar theoretical guarantees. $D^{+} V$ event-based stabilization results in slower convergence but leads to larger inter-execution times than the ISS one which results in faster convergence. Moreover, the first triggering time occurs with $\varphi_{1}$. This is consistent with Proposition 4. For both approaches, $\sigma$

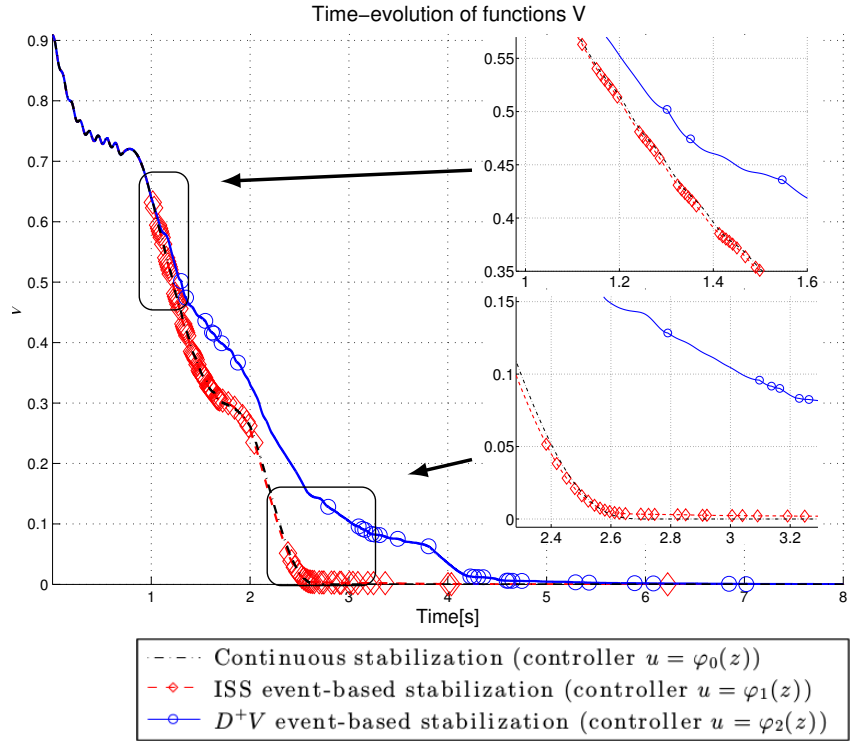

Figure 3. Time-evolution of functions $V$. Legend: Dasheddotted black line for continuous stabilization with controller $u=\varphi_{0}(z)$, dashed red line with red diamond marker for ISS event-based stabilization with controller $u=\varphi_{1}(z)$ and blue line with blue circle marker for $D^{+} V$ event-based stabilization with controller $u=\varphi_{2}(z)$.

has been chosen to reduce as much as possible the number of triggering times. The closer $\sigma$ is to zero, the faster triggering is required. Figure 4 shows the first component of solution when stabilizing with both $\varphi_{1}$ (left) and $\varphi_{2}$ (right). Note that for both approaches, oscillations are presented near the equilibrium and asymptotic stability is achieved. It is worth remarking that under continuous stabilization with $\varphi_{0}$, it is possible to achieve the convergence to the equilibrium in finite time. Such a time, for this particular illustrating example, is given by $T_{F}=\frac{1}{\underline{\lambda}}+\frac{1}{\lambda_{1}}+\frac{1}{\lambda_{2}}=\frac{4+\sqrt{2}}{2} s$.

Moreover, for both event-based approaches, we ran simulations for several initial conditions given by $y_{a, b}^{0}(x)=$ $\left[a x(1-x) \quad \frac{b}{2} \sin ((2 a) \pi x)\right]^{T}, a=1, \ldots, 5$ and $b=1, \ldots, 10$ on a frame of $8 \mathrm{~s}$. Note that these initial conditions satisfy the zero-order compatibility condition. We have computed the duration intervals between two triggering times (inter-execution times). The mean value, standard deviation and the coefficient of variation of interexecution times for both approaches are reported in Table 1 and the density of such inter-execution times is given in Figure 5. The mean value number of trigger-

Table 1

Mean value, standard deviation and variability of interexecution times for ISS event-based stabilization approach $\left(\varphi_{1}\right)$ and $D^{+} V$ event-based stabilization approach $\left(\varphi_{2}\right)$.

\begin{tabular}{|c||c|c|c|}
\hline & Mean value & Standard deviation & Coefficient of variation \\
\hline ISS event-based & 0.0448 & 0.1702 & 3.8024 \\
\hline$D^{+} V$ event-based & 0.1361 & 0.1972 & 1.4489 \\
\hline
\end{tabular}

ing times with $\varphi_{1}$ was 121.1 events whereas with $\varphi_{2}$ was 45.72 events. They both are considerable less with respect to continuous time approach $\varphi_{0}$ since the dis- 

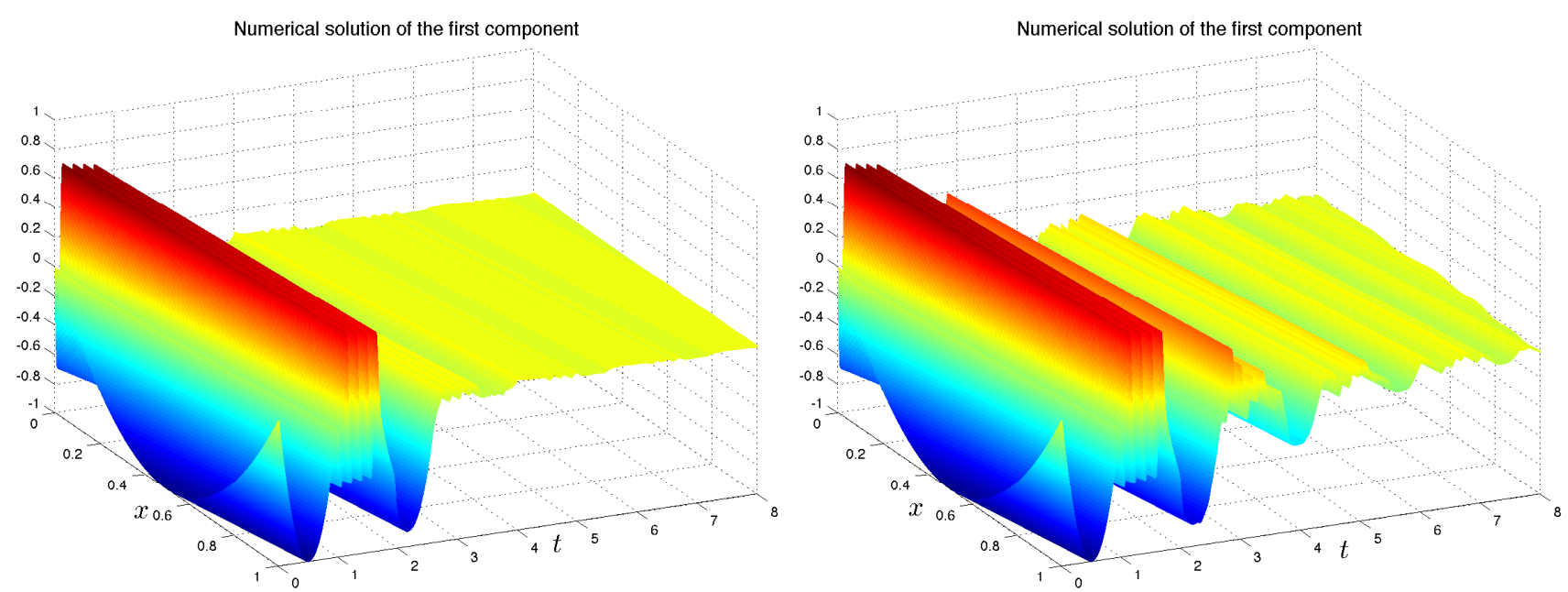

Figure 4. Numerical solution of the first component $y_{1}$ of the closed-loop system with controller $u=\varphi_{1}(z)$ (left) and with controller $u=\varphi_{2}(z)$ (right).
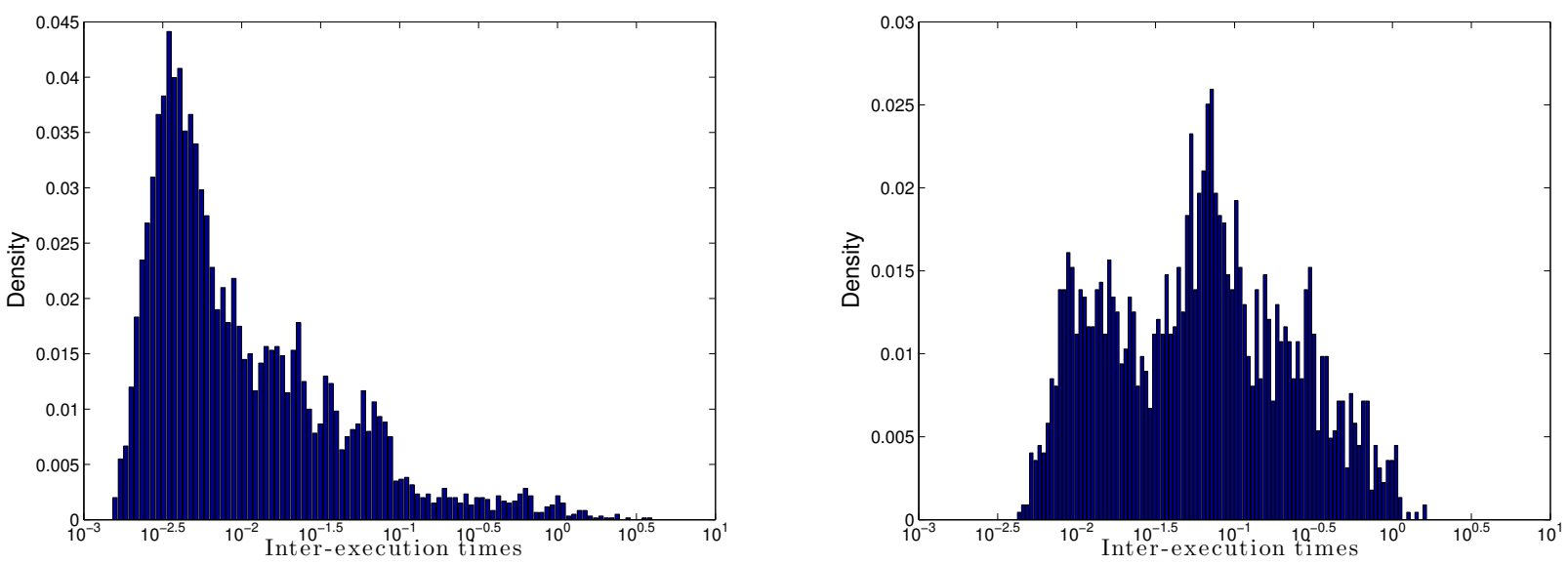

Figure 5. Density of the inter-execution times with controller $u=\varphi_{1}(z)$ (left) and with controller $u=\varphi_{2}(z)$ (right).

cretization time for all simulations was $N T=8000$ with $\Delta t=1 \times 10^{-3}$. It can be seen that using $\varphi_{2}$ results in larger inter-execution times in average than $\varphi_{1}$. In addition, $\varphi_{2}$ reduces the variability of the inter execution times and with $\varphi_{1}$ it is needed to sample faster than with $\varphi_{2}$.

\section{Conclusion}

In this paper, event-based boundary controls to stabilize a linear hyperbolic system of conservation laws have been designed. The analysis of global exponential stability is based on Lyapunov techniques. Moreover, we have proved that under the two event-based stabilization approaches, the solution to the closed-loop system exists and is unique. This paper might be considered as the first contribution to event-based control of Hyperbolic PDEs, and complements the work of [13] and [31] on sampled data control of parabolic PDEs and on event-based control of parabolic PDEs, respectively.
This work leaves some open questions for future works. The event-based stabilization approaches may be applied to a linear hyperbolic system of balance laws. Another interesting point is to apply this control strategy to open channels modeled by the Saint-Venant equations (see e.g. [8]). Indeed, actuation might be expensive due to the actuator inertia when regulating the water level and the water flow rate by using gates opening as the control actions. Then, event-based control would suggest to modulate efficiently the gates opening, only when needed. Another interesting application could be flow control on vehicle highway traffic networks with junctions as considered in [4]. It would generalize what it has been done in Section 4. The rate inflow might be controlled throughout traffic lights modulation in strategies such as ramp-metering on event-based fashion which is actually a realistic approach for the actuator in the system. Concerning the event-based algorithms, it can also be interesting to add an internal dynamic so as it filters 
the event-based signals as it is done in [15] for nonlinear systems. Finally, it could be fruitful to consider performance and rate convergence issues by studying the impact of the event-based control parameters as well as robustness with respect to measurement noise.

\section{Appendix: Proof of Lemma 2}

Let us prove that $V$ is continuous and right differentiable on $\mathbb{R}^{+}$. From (16), it follows that

$$
V(y)=\sum_{d=1}^{n} Q_{d d} V_{d}(y)
$$

where $V_{d}(y)=\int_{0}^{1} y_{d}^{2}(x) e^{-2 \mu x} d x$. Let $y$ be a solution of (1)-(3), let $t$ in $\mathbb{R}^{+}, x \in[0,1], h>0$ then

$$
y_{d}(t+h, x)= \begin{cases}y_{d}\left(t+h-\frac{x}{\lambda_{d}}, 0\right), & \text { if } \lambda_{d} h \geq x \\ y_{d}\left(t, x-\lambda_{d} h\right), & \text { if } \lambda_{d} h<x\end{cases}
$$

Let $V(t)=V(y(t, \cdot))$ and $V_{d}(t)=V_{d}\left(y_{d}(t, \cdot)\right)$. Using (49), $V_{d}(t+h)-V_{d}(t)$ is computed as follows:

$$
\begin{aligned}
V_{d}(t+h)-V_{d}(t)= & \int_{0}^{\lambda_{d} h} y_{d}^{2}\left(t+h-\frac{x}{\lambda_{d}}, 0\right) e^{-2 \mu x} d x \\
& +\int_{\lambda_{d} h}^{1} y_{d}^{2}\left(t, x-\lambda_{d} h\right) e^{-2 \mu x} d x \\
& -\int_{0}^{1} y_{d}^{2}(t, x) e^{-2 \mu x} d x \\
= & \int_{0}^{\lambda_{d} h} y_{d}^{2}\left(t+h-\frac{x}{\lambda_{d}}, 0\right) e^{-2 \mu x} d x \\
& +\int_{0}^{1-\lambda_{d} h} y_{d}^{2}(t, x) e^{-2 \mu x} e^{-2 \mu \lambda_{d} h} d x \\
& -\int_{0}^{1} y_{d}^{2}(t, x) e^{-2 \mu x} d x
\end{aligned}
$$

Hence,

$$
\begin{aligned}
V_{d}(t+h)-V_{d}(t)= & \int_{0}^{\lambda_{d} h} y_{d}^{2}\left(t+h-\frac{x}{\lambda_{d}}, 0\right) e^{-2 \mu x} d x \\
& -e^{-2 \mu \lambda_{d} h} \int_{1-\lambda_{d} h}^{1} y_{d}^{2}(t, x) e^{-2 \mu x} d x \\
& +\left(e^{-2 \mu \lambda_{d} h}-1\right) \int_{0}^{1} y_{d}^{2}(t, x) e^{-2 \mu x} d x
\end{aligned}
$$

Since $y_{d}(\cdot, 0) \in \mathcal{C}_{r p w}\left(\mathbb{R}^{+}, \mathbb{R}^{n}\right)$, we have

$$
\lim _{h \rightarrow 0^{+}} \frac{1}{h} \int_{0}^{\lambda_{d} h} y_{d}^{2}\left(t+h-\frac{x}{\lambda_{d}}, 0\right) e^{-2 \mu x} d x=\lambda_{d} y_{d}^{2}(t, 0)
$$

Now, due to the fact that $y_{d}(t, \cdot) \in \mathcal{C}_{l p w}\left([0,1], \mathbb{R}^{n}\right)$, for all $t \geq 0$,

$\lim _{h \rightarrow 0^{+}} \frac{1}{h} e^{-2 \mu \lambda_{d} h} \int_{1-\lambda_{d} h}^{1} y_{d}^{2}(t, x) e^{-2 \mu x} d x=\lambda_{d} y_{d}^{2}(t, 1) e^{-2 \mu}$
In addition, on gets

$$
\lim _{h \rightarrow 0^{+}} \frac{1}{h}\left(e^{-2 \mu \lambda_{d} h}-1\right)=-2 \mu \lambda_{d}
$$

therefore,

$$
\begin{aligned}
\lim _{h \rightarrow 0^{+}} \frac{V_{d}(t+h)-V_{d}(t)}{h}= & \lambda_{d} y_{d}^{2}(t, 0)-\lambda_{d} y_{d}^{2}(t, 1) e^{-2 \mu} \\
& -2 \mu \lambda_{d} \int_{0}^{1} y_{d}^{2}(t, x) e^{-2 \mu x} d x
\end{aligned}
$$

From (48) and (50) we get,

$$
\begin{aligned}
\lim _{h \rightarrow 0^{+}} \frac{V(t+h)-V(t)}{h}= & \sum_{d=1}^{n} Q_{d d}\left(\lambda_{d} y_{d}^{2}(t, 0)\right. \\
& -\lambda_{d} y_{d}^{2}(t, 1) e^{-2 \mu} \\
& \left.-2 \mu \lambda_{d} \int_{0}^{1} y_{d}^{2}(t, x) e^{-2 \mu x} d x\right)
\end{aligned}
$$

which proves that $V$ is right differentiable and in turn right continuous. Moreover, since $D^{+} V=$ $\lim _{h \rightarrow 0^{+}} \frac{V(t+h)-V(t)}{h},(27)$ holds. In order to prove that $V$ is left continuous, let us consider the case when $h<0$. Then,

$y_{d}(t+h, x)= \begin{cases}y_{d}\left(t+h-\frac{(x-1)}{\lambda_{d}}, 1\right), & \text { if } x \geq \lambda_{d} h+1 \\ y_{d}\left(t, x-\lambda_{d} h\right), & \text { if } x<\lambda_{d} h+1\end{cases}$

and

$$
\begin{aligned}
V_{d}(t+h)-V_{d}(t)= & \int_{-\lambda_{d} h}^{1} y_{d}^{2}(t, x) e^{-2 \mu x} e^{-2 \mu \lambda_{d} h} d x \\
& +\int_{\lambda_{d} h+1}^{1} y_{d}^{2}\left(t+h-\frac{(x-1)}{\lambda_{d}}, 1\right) e^{-2 \mu x} d x \\
& -\int_{0}^{1} y_{d}^{2}(t, x) e^{-2 \mu x} d x
\end{aligned}
$$

which can be rewritten as follows,

$$
\begin{aligned}
V_{d}(t+h)-V_{d}(t)= & \int_{\lambda_{d} h+1}^{1} y_{d}^{2}\left(t+h-\frac{(x-1)}{\lambda_{d}}, 1\right) e^{-2 \mu x} d x \\
& -e^{-2 \mu \lambda_{d} h} \int_{0}^{-\lambda_{d} h} y_{d}^{2}(t, x) e^{-2 \mu x} d x \\
& +\left(e^{-2 \mu \lambda_{d} h}-1\right) \int_{0}^{1} y_{d}^{2}(t, x) e^{-2 \mu x} d x
\end{aligned}
$$

From (52) it can be noticed that,

$$
\lim _{h \rightarrow 0^{-}} V_{d}(t+h)-V_{d}(t)=0
$$

Therefore, $V$ is left continuous. Since it is also right continuous, it is continuous on $\mathbb{R}^{+}$. This concludes the proof of Lemma 2. 


\section{References}

[1] K. J. Åström and B. Bernhardsson. Comparison of periodic and event based sampling for first-order stochastic systems. In Proceedings of the 14th IFAC World congress, volume 11, pages 301-306, 1999 .

[2] G. Bastin, J.-M. Coron, and B. d'Andréa Novel. Using hyperbolic systems of balance laws for modeling, control and stability analysis of physical networks. In Lecture notes for the Pre-Congress Workshop on Complex Embedded and Networked Control Systems, Seoul, Korea, 2008.

[3] G. Bastin, B. Haut, J.-M. Coron, and d'Andréa Novel. Lyapunov stability analysis of networks of scalar conservation laws. Networks and Heterogeneous Media, 2(4):749, 2007.

[4] G. M. Coclite, M. Garavello, and B. Piccoli. Traffic flow on a road network. SIAM Journal on Mathematical Analysis, 36(6):1862-1886, 2005.

[5] J.-M. Coron, G. Bastin, and B. d'Andréa Novel. Dissipative boundary conditions for one-dimensional nonlinear hyperbolic systems. SIAM Journal on Control and Optimization, 47(3):1460-1498, 2008.

[6] J-M Coron, B. d'Andréa Novel, and G. Bastin. A strict Lyapunov function for boundary control of hyperbolic systems of conservation laws. IEEE Transactions on Automatic Control, 52(1):2-11, 2007.

[7] J.-M. Coron, R. Vazquez, M. Krstic, and G. Bastin. Local exponential $H^{2}$ stabilization of a $2 \times 2$ quasilinear hyperbolic system using backstepping. SIAM Journal on Control and Optimization, 51(3):2005-2035, 2013.

[8] J. de Halleux, C. Prieur, J.-M. Coron, B. d'Andréa Novel, and G. Bastin. Boundary feedback control in networks of open channels. Automatica, 39(8):1365-1376, 2003.

[9] A. Diagne, G. Bastin, and J.-M. Coron. Lyapunov exponential stability of 1-D linear hyperbolic systems of balance laws. Automatica, 48(1):109-114, 2012

[10] S.M. Djouadi, R.C. Camphouse, and J.H. Myatt. Reduced order models for boundary feedback flow control. In American Control Conference, pages 4005-4010, Seattle, Washington, 2008.

[11] V. Dos Santos, G. Bastin, J-M. Coron, and B. d'Andréa Novel. Boundary control with integral action for hyperbolic systems of conservation laws: Stability and experiments. Automatica, 44(5):1310-1318, 2008.

[12] S. Durand, N. Marchand, and J. F. Guerrero Castellanos. Event-based stabilization of nonlinear time-delay systems. In 19th IFAC World Congress, Cape Town, South Africa, 2014.

[13] E. Fridman and A. Blighovsky. Robust sampled-data control of a class of semilinear parabolic systems. Automatica, 48(5):826-836, 2012.

[14] E. Fridman and Y. Orlov. An LMI approach to $H \infty$ boundary control of semilinear parabolic and hyperbolic systems. Automatica, 45(9):2060-2066, 2009.

[15] A. Girard. Dynamic triggering mechanisms for eventtriggered control. IEEE Transactions on Automatic Control, 60(7):1992-1997, July 2015.

[16] M. Gugat, M. Dick, and G. Leugering. Gas flow in fan-shaped networks: Classical solutions and feedback stabilization. SIAM Journal on Control and Optimization, 49(5):21012117, 2011.

[17] F.M. Hante, G. Leugering, and T.I. Seidman. Modeling and analysis of modal switching in networked transport systems. Applied Mathematics and Optimization, 59(2):275-292, 2009.
[18] W.P.M.H. Heemels, K.H. Johansson, and P. Tabuada. An introduction to event-triggered and self-triggered control. In Proceedings of the 51st IEEE Conference on Decision and Control,2012, pages 3270-3285, Maui, Hawaii, 2012.

[19] M. Krstic and A. Smyshlyaev. Backstepping boundary control for first-order hyperbolic PDEs and application to systems with actuator and sensor delays. Systems $\&$ Control Letters, 57(9):750-758, 2008.

[20] P.-O. Lamare, A. Girard, and C. Prieur. Switching rules for stabilization of linear systems of conservation laws. SIAM Journal on Control and Optimization, 53(3):1599-1624, 2015.

[21] M. Lemmon. Event-triggered feedback in control, estimation, and optimization. In Networked Control Systems, pages 293358. Springer, 2010.

[22] T.-T. Li. Global Classical Solutions for Quasi-Linear Hyperbolic Systems. Research in Applied Mathematics. Masson and Wiley, 1994.

[23] J. Lofberg. Yalmip: A toolbox for modeling and optimization in MATLAB. In IEEE International Symposium on Computer Aided Control Systems Design, 2004, pages 284289, Taipei, Taiwan, 2004.

[24] N. Marchand, S. Durand, and J. F. G. Castellanos. A general formula for event-based stabilization of nonlinear systems. IEEE Transactions on Automatic Control, 58(5):1332-1337, 2013.

[25] G. Martin and H. Michaël. Existence of classical solutions and feedback stabilization for the flow in gas networks. ESAIM, Control Optim. Calc. Var., 17(1):28-51, 2011.

[26] Y Orlov. Discontinuous systems: Lyapunov analysis and robust synthesis under uncertainty conditions. SpringerVerlag, 2009.

[27] R. Postoyan, P. Tabuada, D. Nesic, and A. Anta. A framework for the event-triggered stabilization of nonlinear systems. IEEE Transactions on Automatic Control, 60(4):982-996, 2015.

[28] C. Prieur, A. Girard, and E. Witrant. Stability of switched linear hyperbolic systems by Lyapunov techniques. IEEE Transactions on Automatic Control, 59(8):2196-2202, 2014.

[29] C. Prieur and F. Mazenc. ISS-Lyapunov functions for timevarying hyperbolic systems of balance laws. Mathematics of Control, Signals, and Systems, 24(1-2):111-134, 2012.

[30] C. Prieur, J. Winkin, and G. Bastin. Robust boundary control of systems of conservation laws. Mathematics of Control, Signals, and Systems, 20(2):173-197, 2008.

[31] A. Selivanov and E. Fridman. Distributed event-triggered control of transport-reaction systems. In 1st IFAC Conference on Modelling, Identification and Control of Nonlinear Systems, volume 48, pages 593-597, Saint Petersburg, Russia, 2015.

[32] L.F Shampine. Solving hyperbolic PDEs in MATLAB. Applied Numerical Analysis \& Computational Mathematics, 2(3):346-358, 2005.

[33] L.F. Shampine. Two-step Lax-Friedrichs method. Applied Mathematics Letters, 18(10):1134-1136, 2005.

[34] P. Tabuada. Event-triggered real-time scheduling of stabilizing control tasks. IEEE Transactions on Automatic Control, 52(9):1680-1685, 2007.

[35] Z. Yao and N.H. El-Farra. Resource-aware model predictive control of spatially distributed processes using event-triggered communication. In Proceedings of the 52nd IEEE Conference on Decision and Control, pages 3726-3731, Florence, Italy, 2013.

[36] K.-E. Årzén. A Simple Event-based PID Controller. In Proc. 14th World Congress of IFAC, Beijing, pages 423-428, 1999. 